1

\title{
PRINCIPLES OF METABOLIC CONTROL
}

\author{
William C. Plaxton
}

\section{KEY CONCEPTS}

The ability to control the rates of metabolic processes in response to changes in the internal or external environment is an indispensable attribute of living cells that must have arisen with life's origin. This adaptability is necessary for conserving the stability of the intracellular environment (homeostasis), which is, in turn, essential for maintaining an efficient functional state. In the absence of such control, all metabolic processes would achieve a state of equilibrium with the external environment. For example, the intracellular storage of a fuel macromolecule such as glycogen would be impossible since there is an enzyme (glycogen phosphorylase) dedicated to catalyzing the breakdown of this storage polyglucan into its constituent glucosyl units. Obviously, the existence of glycogen as an important energy store in animal tissues implies that the activity of glycogen phosphorylase is carefully controlled so as to allow this fuel to be utilized as dictated by the needs of the organism. Indeed, elaborate regulatory mechanisms have been discovered that affect glycogen phosphorylase activity, thereby allowing wide variations in the rate of glycogen breakdown in vivo. The aim of this chapter is to outline the biochemical regulatory mechanisms that are believed to be the most important in metabolic control. Practical aspects for the study of metabolism and its control, as well as the advantages and disadvantages of qualitative versus quantitative approaches to metabolic control, will also be highlighted.

\section{Metabolic Renaissance in Postgenome Era?}

The remarkable advances in molecular genetics that have occurred over the past couple of decades have somewhat eclipsed areas of traditional biochemistry such as protein chemistry, enzymology, and metabolic control. With many genomes sequenced and others nearing completion, the next step is the less straightforward task of analyzing the expression and function of gene products (proteins), as well as more thoroughly elucidating metabolism and its control. The task of completing the picture of all cellular proteins, their actions and reactions, is one of the biggest challenges facing life science researchers today. Although molecular biology has generated many impressive techniques [e.g., protein overexpression, site-directed mutagenesis, metabolic engineering, complementary deoxyribonucleic acid (cDNA) microarrays, etc.] for assessing various aspects of protein/enzyme structure-function and regulatory control, one cannot deduce the properties of a functional protein or the kinetic and regulatory properties of an enzyme solely from genetic information. Moreover, recent animal, plant, and microbial genome sequencing projects have revealed a plethora of gene sequences that encode proteins having unknown functions. Furthermore, many organisms whose genomes have been sequenced have not had their metabolism extensively studied. Where feasible, their metabolic phenotype is determined using annotated genome sequence data. Thus, there appears to be a resurgence of interest in protein, enzymological, and metabolic research for understanding biological processes in the postgenome era. Efficient approaches are needed for determining: (a) the function of unknown gene products, (b) protein expression in different cells under various conditions, (c) covalent modifications of proteins in response to different stimuli, (d) protein-protein interactions, (d) the relationship between protein structure and protein function, and (e) the sophisticated mechanisms that serve to control the flux of metabolites through specific

Functional Metabolism: Regulation and Adaptation, edited by Kenneth B. Storey. ISBN 0-471-41090-X Copyright (C) 2004 John Wiley \& Sons, Inc. 
metabolic pathways in vivo. Novel methods are also being developed to map proteomes (i.e., the proteins encoded by the genome) and to discover new enzymes of interest.

\section{Metabolic Engineering}

Since it is now possible to manipulate nucleic acids and gene expression at will, an important goal of biotechnology is to modify (usually enhance) the output of specific biosynthetic pathways via the process of metabolic engineering. Contemporary genetic engineering techniques have created the potential to directly modify the metabolism of a target organism in a desired fashion. However, the ability to manipulate an organism's genetics has thus far transcended our ability to predict the effects of these manipulations on metabolism. Our lack of a thorough appreciation for, and understanding of, metabolic control is reflected by the meager results from most attempts to apply the powerful tools of molecular biology and genetic transformation to the practical goal of metabolic engineering. Many unsuccessful efforts at so-called shotgun metabolic engineering have been based upon the misguided assumption that if the expression of a gene encoding a particular enzyme is suitably manipulated, then there will be a corresponding change in both the in vivo activity of the encoded enzyme as well as the flux (or rate of movement) of metabolites through the pathway in which the enzyme functions. "Rational" metabolic engineering is a longerterm, but arguably more scientific and interesting process that involves the targeted and purposeful alteration of a specific metabolic pathway. It does not necessarily depend upon altering the concentration of an enzyme but could be carried out by introducing a mutant (or heterologous) enzyme with altered control properties. Apart from an ability to manipulate nucleic acids, rational metabolic engineering also requires a strong background in protein/enzyme and metabolic biochemistry.

\section{Metabolic Regulation versus Metabolic Control}

Although biochemists frequently employ the terms regulation and control interchangeably, the need to discriminate between these terms has been emphasized (Fell, 1997). Metabolic control refers to adjusting the output of a metabolic pathway in response to an external signal. By contrast, metabolic regulation occurs when an organism maintains some variable relatively constant over time, despite fluctuations in external conditions. Homeostasis is therefore a consequence of metabolic regulation, which itself may be a result of metabolic control. For example, the regulation of mammalian blood glucose is largely due to the secreted peptide hormones glucagon ("starved" signal) and insulin ("fed" signal) controlling intracellular metabolism within the liver. In this case, the concentration of blood glucose is regulated (kept constant) mainly by controlling (varying) fluxes of metabolic pathways (i.e., glycogen breakdown versus synthesis, glycolysis, gluconeogenesis) in hepatocytes. Regulation and control are properties of highly elaborate metabolic systems. An ongoing challenge is to link our knowledge of molecular, reductionist-based, enzyme control mechanisms to organism-level explan-ations of metabolic regulation.

\section{Complexity of Metabolism and Concept of Biochemical Unity}

As protein catalysts, enzymes vastly accelerate the rates of chemical reactions without themselves undergoing a permanent change. As each cellular reaction is catalyzed by its own enzyme, every cell contains a large number of different enzymes. Although a "simple" prokaryote, such as Escherichia coli, is only about $1 / 500$ th the size of a typical eukaryotic cell, each $E$. coli cell contains about 3000 different proteins, at least $90 \%$ of which are enzymes. The metabolic complexity of all cells is reflected by the many separate enzymatic reactions that make up the metabolic pathways that collectively constitute metabolism.

Despite its complexity, a general understanding of metabolism has been achieved because common solutions to the problem of biochemical design have been evolved. Thus, the types of substrates, cosubstrates, cofactors, fuels, and types of metabolic pathways used are common to most cells. This is the concept of biochemical unity. In general, biochemical unity also applies to metabolic regulation and metabolic control. Comparative biochemistry has revealed that the types of control mechanisms found in metabolic pathways are similar from one organism to the next. However, it is the implementation of these designs - the regulatory details-that cannot only differ widely from species to species but can differ widely for similar metabolic pathways in different cell types of a single organism, or even within different organelles of a single cell.

For example, citrate synthase, which catalyzes the reaction acetyl-CoA + oxaloacetate $\rightarrow$ citrate $+\mathrm{CoA}$, is controlled in dissimilar manners in different cells. In respiring animal cells, a major function for this enzyme is in the citric acid cycle that operates in the mitochondria in conjunction with oxidative phosphorylation to produce adenosine $5^{\prime}$-triphosphate (ATP). Here, the overall end product, ATP, feeds back to inhibit citrate synthase. This regulatory mechanism is logical since at high ATP levels, the ATPgenerating citric acid cycle will then be inhibited, but if ATP levels fall, substrate catabolism by the cycle will speed up. In E. coli, however, citrate synthase and the citric acid cycle have a different function. This bacterium lives a mainly anaerobic life, generating its ATP primarily 
via the fermentation of glucose by glycolysis. The main role of the citric acid cycle in E. coli is in the generation of biosynthetic precursors and reducing power [reduced nicotinamide adenine dinucleotide (NADH)]. E. coli citrate synthase is unaffected by ATP, but it is inhibited by one of the ultimate end products of the cycle, NADH. Germinating seeds contain a third type of citrate synthase, localized in the glyoxysome. This isozyme is insensitive to both ATP and $\mathrm{NADH}$, and here the enzyme functions as part of the glyoxylate cycle, an indispensable component in the metabolic conversion of fatty acids derived from storage triacylglycerides into sucrose. Thus, the concept of biochemical unity tends to break down when individual metabolic controls are compared. Although the structure and products of a metabolic enzyme or a metabolic pathway may be identical in various organisms, the environment and function of that enzyme or pathway may not be the same. Nevertheless, all metabolic controls have a common basis, and certain regulatory strategies are ubiquitous.

\section{BASIS OF METABOLIC CONTROL}

\section{Pacemaker Enzymes}

It is self-evident that the flux of metabolites through any pathway must be closely coordinated with the needs of the cell, tissue, or organism for the final end product(s) of the pathway. The traditional view of metabolic control is that such regulation is accomplished by altering the activity of at least one pacemaker enzyme (or rate-determining step) of the pathway. Thus, a major focus of enzymology has been to characterize these key enzyme reactions-the pacemakers - that are believed to be most important in controlling pathway flux. Substantial efforts have been directed to identifying the pacemaker enzyme(s) of metabolic pathways, as well as the complex mechanisms that serve to modulate the activities of these key enzymes.

\section{Identification of a Pacemaker Enzyme}

Normally, the pacemaker enzyme(s) of a pathway has a low activity overall, is subject to control by metabolites other than its substrates, and is often positioned as the first committed step of a pathway, directly after major branch points, or at the last step of a "multi-input" pathway. However, such circumstantial evidence for a putative pacemaker enzyme-catalyzed reaction still requires confirmation by techniques that rely upon accurate quantification of the in vivo concentrations of the enzyme's substrate(s) and product(s).

The standard method for determining metabolite concentrations begins with ultrafast freezing of cells/tissues in liquid nitrogen at $-196^{\circ} \mathrm{C}$. This effectively quenches any reactions that could lead to artifactual alterations in metabolite levels. The frozen tissue is then extracted by homogenization at low $\mathrm{pH}$ in cold perchloric acid, which inactivates enzymes that would affect the metabolites of interest. Following centrifugation to remove cell debris and precipitated proteins, the supernatant is neutralized and analyzed for metabolites by appropriate enzymatic or chemical methods. More recently, ${ }^{31} \mathrm{P}$ nuclear magnetic resonance $\left({ }^{31} \mathrm{P}-\mathrm{NMR}\right)$ has become widely used for determining the intracellular concentrations of phosphate-containing metabolites such as the hexose-phosphates, adenylates, phosphocreatine, and inorganic phosphate. It is now feasible to measure the concentrations of phosphate-containing metabolites in a tissue, perfused organ, or even an intact living organism inserted into the widebore magnet of a NMR spectrometer. Results obtained from ${ }^{31} \mathrm{P}-\mathrm{NMR}$ are generally consistent with those obtained by acid extraction techniques. However, noninvasive NMR techniques continue to hold great promise for providing a detailed insight into metabolite levels of living cells, and how these levels may vary following perturbations such as oxygen deprivation, or the addition of metabolites or hormones. Whether obtained via classical or NMR methods, the amount of a metabolite (determined as micromoles per gram of tissue), can also be expressed as concentration [i.e., in millimolars $(\mathrm{mM})$ ] in the intracellular water if the water content of the tissue is known.

Accurate determinations of metabolite levels can be difficult, particularly if the same metabolite is distributed between several intracellular compartments. Nevertheless, the availability of metabolite data is quite relevant to determining how and where flux control of a specific metabolic pathway is exerted, that is, probable pacemaker enzyme(s). A major controlling enzyme should theoretically catalyze the slowest step in the pathway. Thus, a reaction that is far from equilibrium in vivo is likely to be catalyzed by a pacemaker enzyme (although under certain conditions, enzymes that catalyze reactions close to equilibrium may also be regulatory). The reason that equilibrium is not achieved is that owing to the action of various "fine" metabolic controls (such as feedback allosteric inhibition; see below) there is insufficient active enzyme present to bring the reaction to equilibrium in vivo. Hence, a metabolic biochemist interested in identifying important sites of pathway control initially searches for those enzymes that catalyze nonequilibrium reactions in vivo (i.e., catalyze irreversible reactions that have a highly negative overall free energy change). This requires measurement of the intracellular concentrations of the particular metabolites involved. Results obtained with a rat heart perfused with glucose are listed in Table 1.1.

For the glycolytic reaction catalyzed by 6-phosphofructokinase (PFK): F6P + ATP $\rightarrow$ FBP + ADP, the ratio of its intracellular concentrations of products : substrates, known 
TABLE 1.1 Intracellular Concentration of Several Metabolites Obtained with a Rat Heart Perfused with Glucose

\begin{tabular}{lc}
\hline Metabolite & $\begin{array}{c}\text { Intracellular Concentration } \\
(\mathrm{mM})\end{array}$ \\
\hline D-Fructose-6-phosphate (F6P) & 0.09 \\
D-Fructose-1,6-bisphosphate (FBP) & 0.02 \\
ATP & 11.5 \\
ADP & 1.3 \\
AMP & 0.17 \\
\hline
\end{tabular}

Source: Price and Stevens (1989).

as the mass action ratio $(\Gamma)$, is given by

$$
\Gamma=\frac{[\mathrm{FBP}][\mathrm{ADP}]=0.025}{[\mathrm{~F} 6 \mathrm{P}][\mathrm{ATP}]}
$$

where F6P is fructose-6-phosphate, FBP is fructose-1,6biphosphate and ADP is adenosine $5^{\prime}$-diphosphate. The equilibrium constant $\left(K_{\text {eq }}\right)$ for a chemical reaction, a value that is independent of the presence or absence of a catalyst (enzyme), can be determined in separate experiments under physiological $\mathrm{pH}$, temperature, and pressure. The $K_{\text {eq }}$ for the aforementioned reaction is about 1200 . Since this value is 48,000 -fold greater than the reaction's corresponding $\Gamma$ value in perfused rat heart, it is evident that the reaction in vivo is very far removed from equilibrium and, thus, is essentially irreversible. Therefore, PFK is regarded as a probable pacemaker enzyme of the glycolytic pathway. This is logical because PFK catalyzes the first committed step of glycolysis, that is, the first step that does not form part of other metabolic processes as well. By contrast, for the reaction catalyzed by adenylate kinase: ATP + AMP $\leftrightarrow 2 \mathrm{ADP}$,

$$
\Gamma=\frac{[\mathrm{ADP}]^{2}}{[\mathrm{ATP}][\mathrm{AMP}]}=0.85
$$

where AMP is adenosine $5^{\prime}$-monophosphate. This is very close to the reaction's $K_{\text {eq }}$ value of 0.44 . This indicates that rat heart adenylate kinase is sufficiently active in vivo to allow the reaction to remain very close to equilibrium (or readily reversible).

Another method used to identify pacemaker enzyme(s) that has been employed is the "crossover theorem," which states that when pathway flux is enhanced, the substrate concentration of the pacemaker enzyme will decrease and its product concentration will increase (and vice versa for a flux decrease). A crossover in relative metabolite levels between two physiological states indicates where a regulatory signal has acted on a pacemaker enzyme to alter flux. For example, in yeast, as well as in many animal and plant tissues, oxygen deprivation greatly enhances glycolytic flux (owing to the need to generate ATP via anaerobic fermentation). This is associated with an immediate reduction in intracellular [F6P] and an increase in [FBP]. This results in a "positive crossover" between F6P and FBP and indicates that the perturbation (anoxia) has activated PFK in vivo so that the concentration of its substrate, F6P, is lowered whereas that of its product, FBP, is increased. An elevated [FBP] would directly cause an increased flux through the next enzyme in the glycolytic pathway, FBP aldolase (which catalyzes a reaction close to equilibrium and shows no crossover following the aerobic to anoxic transition). This flux increase would then be transmitted to the remainder of the glycolytic sequence. Crossover analysis clearly identifies PFK as a pacemaker enzyme whose activity can respond appropriately to facilitate the marked stimulation of glycolysis that accompanies the imposition of anoxia stress. This example also underscores an important principle in metabolic biochemistry research: namely, that the full elucidation of pathway control is often dependent on a thorough comparative analysis of a control versus perturbed (i.e., stressed) tissue. Correlation of alterations in metabolic fluxes and metabolite levels that ensue from a perturbation of cells/tissues generates indispensable information for the metabolic biochemist seeking to identify key sites of control in a particular pathway.

\section{Enzyme Purification}

From the various approaches described above it is possible to pinpoint the most important sites of control of a metabolic pathway. The next step is to examine the molecular and kinetic properties of the putative pacemaker enzyme(s), particularly those properties that might be involved in the control of pathway flux. Of particular interest are the following questions:

1. What is the enzyme's subunit structure? Pacemaker enzymes are invariably multimeric; that is, in their native state they consist of two or more subunits held together by noncovalent bonds. A more complex protein structure appears to be a prerequisite for a more complex protein function/regulation (i.e., allosterism, etc.).

2. How does the activity of the enzyme vary with alterations in assay $\mathrm{pH}$ and substrate(s) concentration(s)? Are hyperbolic (Michaelis-Menten) or sigmoidal (cooperative) substrate saturation kinetics observed? As discussed below, the sigmoidal substrate saturation plot observed for some pacemaker enzymes implies that over a certain range of substrate concentrations, the activity is more sensitive to 
[S] than would be the case for enzymes that display hyperbolic kinetics.

3 . Is the activity of the enzyme controlled by any metabolites (referred to as effectors) that are structurally distinct from its own substrate(s) or product(s)? If so, (a) what is the nature of the interaction (i.e., activation or inhibition) and (b) do these metabolite effectors significantly alter enzyme activity in vitro at concentrations that are known to exist in vivo?

4. Is the activity of the enzyme subject to control by covalent modification such as by reversible protein phosphorylation, and if so, what mechanisms serve to control the activities of the modifying enzymes that catalyze these changes?

It is clear that if we hope to gain a detailed understanding of the behavior of an enzyme in a complex biological system (such as an isolated organelle, intact cell, or entire organism) we must first attempt to understand its properties in as simple a system as possible. Thus, one cornerstone of metabolic biochemistry research has been the reductionist approach of enzyme purification and characterization. Enzyme purification eliminates metabolites and contaminating proteins that would otherwise confound kinetic and/or structural studies. By performing accurate and detailed analyses of the kinetic and regulatory properties of purified key enzymes in vitro and then combining these with knowledge of the enzyme's subcellular localization and of the in vivo concentrations of the enzyme's substrates, products, and metabolite effectors, one attempts to formulate a theory for the control of the enzyme in vivo. Such information can have broad practical applications and may be used to provide insights about various metabolic diseases, facilitate the identification of suitable targets for the disruption of a pathogen's metabolism with drugs, or generate key information for biotechnologists wishing to manipulate specific metabolic pathways via metabolic engineering. From in vitro studies of a purified enzyme we can also learn about its structure, specificity for substrates, and reaction mechanism. With sufficient quantities of purified enzyme, structural biochemists can employ powerful methods such as chemical modification and covalent labeling, peptide isolation and sequencing, X-ray crystallography, mass spectroscopy, NMR spectroscopy, and so on, to determine the relationship between an enzyme's structure and its function. This leads to the identification of key amino acid residues that are critical in substrate/product or allosteric effector binding, catalysis and covalent modification (i.e., phosphorylation) sites. Integration of biochemical (kinetic and structural data) and genetic information for a given enzyme ultimately provides a logical basis for site-directed mutagenesis by suggesting which amino acid(s) should be modified to produce a mutant enzyme with altered kinetic/regulatory properties.

\section{Cautionary Note about Relating In Vitro Kinetic Studies of a Purified Enzyme to Its In Vivo Function and Control}

The traditional approach to metabolic control outlined above is largely based upon the extrapolation of the in vitro kinetic/regulatory characteristics of purified enzymes to conditions within the intact cell. However, it is well-known that large multimeric regulatory enzymes such as PFK are susceptible to artefactual posttranslational modifications such as partial proteolysis by endogenous proteases (during purification), as well as dilution-dependent alterations in their oligomeric structure. Even very minor proteolytic clipping may obscure the allosteric properties of a purified regulatory enzyme, without markedly influencing its $V_{\max }$. The diagnosis and prevention of unwanted proteolysis, through the addition of appropriate protease inhibitors to purification buffers, should be a major concern of all enzymologists.

The influence of protein concentration must also be considered because enzymes are present in vivo at far greater concentrations than they are during in vitro kinetic assays. Concentration dependence is believed to be particularly significant for enzymes important in metabolic control because their structure, and hence kinetic/regulatory properties, may be affected by protein-protein interactions. The interactions between enzyme subunits that normally exist at the high protein concentration prevailing in vivo can be specifically promoted in vitro by the addition of molecular crowding agents such as $10 \%(\mathrm{v} / \mathrm{v})$ glycerol or $5 \%(\mathrm{w} / \mathrm{v})$ polyethylene glycol to the reaction mixture. The mechanism involves exclusion of the protein from the aqueous solvent, thus increasing local enzyme concentration and favoring protein-protein interactions. The in vitro activity of many regulatory oligomers, including rat liver PFK, can be enhanced by the presence of such molecular crowding agents and can aid the examination of the catalytic and regulatory properties of enzymes in an in vitro environment that may be a closer approximation of the conditions prevailing in vivo.

\section{Compartmentation of Metabolism}

In the intact cell the individual enzymes of a metabolic pathway function to convert a starting material to end product(s) without necessitating the accumulation of elevated concentrations of the corresponding metabolic intermediates. In addition, there are many connections between the major metabolic pathways, with selected substrates, cofactors, regulatory molecules, and occasionally enzymes being common to more than one pathway. These complex 
interactions can only be fully understood when, in addition to studying the isolated enzymes, some knowledge has been acquired concerning the intracellular location and concentrations of the enzyme and metabolites involved, and of any permeability barriers that separate the individual components. Thus, the functions of many enzymes cannot be fully understood without knowledge of their subcellular location.

The appearance of eukaryotic cells during evolution was associated with the process of compartmentation of metabolism through the formation of specialized organelles such as lysosomes, mitochondria, and plastids, separated from the cytosol and from each other by selectively permeable membranes. Compartmentation concentrates enzymes of a metabolic pathway and their associated metabolites, and prevents the simultaneous occurrence of potentially incompatible metabolic processes. The integration of cellular metabolism necessitates controlled interactions between pathways sequestered in the various subcellular compartments. This is facilitated by the existence of numerous membrane transporter proteins that selectively translocate specific metabolites between subcellular compartments. Thus, a major advance in the biochemical study of eukaryotic cells was the development of methods for separating intact organelles from the cytosol and from each other. This has not only allowed analysis of the overall biological functions of isolated organelles (i.e., respiration in mitochondria, photosynthesis in chloroplasts, etc.) but has also facilitated the determination of the distribution of enzymes and metabolites within cells, as well as the various metabolite translocators of the organelle membranes. Understanding these transport processes is of great importance for the overall understanding of metabolism and its control.

\section{Formulation of a Theory of Metabolic Control}

Once the controlling enzyme(s) of a given pathway have been identified and their kinetic/regulatory properties and subcellular localization investigated, it should be feasible to postulate a theory for the control of pathway flux. The theory should give rise to predictions that can be tested experimentally. This often necessitates collecting data about the activities and state of covalent modification (i.e., phosphorylation status) of the key enzymes, and the concentrations of their substrates and effectors under a variety of physiological conditions. It is important to demonstrate that these parameters reflect the in vivo situation as closely as possible. For example, is the in vivo ratio of a pacemaker enzyme's [activators]: [inhibitors] proportional to pathway flux (i.e., following pathway stimulation, do the levels of the enzyme's activators and inhibitors, respectively, increase and decrease, and vice versa)? Similarly, are the variations in the in vivo concentrations of effectors sufficient to account for the observed changes in enzyme activity or pathway flux? If not, then the initial theory must be revised and retested. This may require a more detailed investigation of the properties of the isolated enzyme.

One problem with the traditional pacemaker approach to metabolic control is that the studies are for the most part qualitative rather than quantitative. For example, the demonstration that a particular enzyme catalyzes a nonequilibrium reaction in vivo, shows a positive crossover during pathway activation, and exhibits pronounced control properties in vitro provides a unequivocal indication that it is relatively important in controlling pathway flux in vivo. However, designation of an enzyme as a pacemaker is not based upon any direct measurement of the precise contribution of each enzyme in a pathway to the overall control of pathway flux. Where more than one enzyme appears to be "regulatory," there is no estimate as to their relative contributions, or how the degree of control exerted by each enzyme might vary under differing physiological circumstances. Furthermore, biological systems may display regulatory properties that are not possessed by their isolated components. In other words, the properties of biological systems tend to be greater than the sum of the properties of their isolated parts (i.e., the so-called Humpty-Dumpty principle). For instance, it would be impossible to understand how a mitochondrion functions in respiration by only studying purified mitochondrial enzymes and electron transport proteins in isolation from each other and from mitochondrial membranes. Thus, another important approach to the problem of metabolic control is to analyze the whole system.

\section{METABOLIC CONTROL ANALYSIS}

The metabolic control analysis (MCA) theory developed by Kacser in 1973 attempts to provide a quantifiable mechanism for probing intact biological systems and interprets the resulting data without preconceived notions as to which enzymes in a pathway are rate-determining steps or pacemakers (Fell, 1997). In fact, an important tenet of MCA theory is that metabolic control is shared among many, if not all, steps in a pathway. As discussed in more detail in a later chapter (and in references at the end of this chapter), Kacser has established the concept of the flux control coefficient $\left(C_{E}^{J}\right)$ whose value specifies the change in metabolic flux $(\Delta J)$ that results from a small change in the activity of any enzyme $(\Delta E)$ in the metabolic system as follows: $C_{E}^{J}=(\Delta J / \Delta E)$. For a linear pathway, the flux control coefficients of the component enzymes will lie between 0 and 1 , with higher values indicating a greater contribution to metabolic control. 
However, for complex pathways involving branches or substrate cycles, the flux control coefficients can hold any value, less than 0 or greater than 1 . It should be emphasized that measurement of a flux control coefficient for a single step in a metabolic pathway may be difficult to interpret. The most satisfactory, yet highly challenging, way to apply MCA to a pathway would be to estimate the flux control coefficients for each component enzyme, as values for a single step may mislead.

Experimental determination of the magnitude of appropriate flux control coefficients apparently yields an unambiguous evaluation of the existing quantitative allotment of control among the various steps in a pathway, under specified conditions. Moreover, since the values of flux control coefficients can redistribute between enzymes according to physiological circumstances, any particular flux control coefficient applies only to the physiological state in which it was determined. Because metabolic control theory predicts that all enzymes in a pathway exert some control on pathway flux, all enzymes (in a linear pathway) should theoretically have flux control coefficients greater than 0 . However, no single enzyme would be expected to have a flux control coefficient as high as 1.0 (which would be the case if pathway flux were entirely controlled by a single pacemaker enzyme). In fact, the summation theorem states that the sum of flux control coefficients for all component enzymes of a metabolic system should equate to 1.0 .

The magnitude of any one flux control coefficient is not an intrinsic property of the enzyme per se but is a system property that depends upon the concurrent activities of all the other enzymes in the pathway. Thus, the value of a flux control coefficient cannot be determined by considering the properties of a purified enzyme since the characteristics and amounts of other enzymes in the system will influence the result. Individual flux control coefficients must therefore be determined experimentally from the intact system by measuring how pathway flux changes following alterations of the activity of a specific enzyme in situ. Advances in molecular biology now allow for direct manipulations of in vivo enzyme activities and continue to yield new information on the control of metabolism. There are a number of excellent publications and several Internet sites devoted to the quantitative MCA approach to metabolic control (see references). The various formulations and concepts of the mathematical models of MCA have given rise to considerable debate over the meaning and usefulness of flux control coefficients (see Text Box 1.1). However, most metabolic biochemists would likely agree that flux control of a metabolic pathway is generally dominated by a minority of its component enzymes (i.e., the pacemakers), although under different physiological conditions the degree of control exerted by the individual enzymes may vary.

\section{TEXT BOX 1.1 THE PFK PARADOX}

Phosphofructokinase (PFK) is generally considered to be an important pacemaker enzyme of the glycolytic pathway. It catalyzes the first unique step of glycolysis, a nonequilibrium reaction in vivo and shows a strong positive crossover concomitant with glycolytic stimulation. PFK is a multimeric enzyme that displays sigmoidal substrate (F6P) saturation kinetics as well as complex and potent allosteric regulation by numerous effectors, the levels which are controlled by the hormonal and/or nutritional status of the tissue. For example, the role of fructose-2,6-bisphosphate as a potent allosteric activator of animal and yeast PFK is wellestablished. However, the use of molecular genetic techniques for the selective overexpression of PFK in yeast, mammals, and plants has failed to yield significant increases in glycolytic flux or respiration that were expected. It appears that the elevated PFK concentration was somewhat compensated for in vivo by changes in the levels of PFK allosteric activators and inhibitors. PFK flux control coefficients were determined to be very small, leading to the surprising conclusion that PFK exerts very little or no control over glycolytic flux or respiration in vivo. Although proponents of MCA have challenged the traditional concept that PFK is a pacemaker enzyme of glycolysis, several MCA advocates have nevertheless agreed that there is little doubt that "control of PFK activity plays a part in glycolytic flux control" (Thomas and Fell, 1998) and "PFK makes an important contribution to the control of glycolysis in most cells" (CornishBowden, 1999). A possible explanation for this "PFK paradox," is that MCA also indicated that significant flux control of glycolysis and respiration lies in the metabolism of key feedback inhibitors of PFK, namely ATP and citrate in yeast and mammalian cells and phosphoenolpyruvate in plant cells. This would suggest the somewhat contradictory conclusion that, although the flux control coefficient for PFK may be low, it does indeed play an important role in the control of carbohydrate catabolism in most cells.

\section{MECHANISMS OF METABOLIC CONTROL}

The magnitude of metabolite flux through any metabolic pathway will depend upon the activities of the individual enzymes involved. It is possible to group mechanisms of metabolic control into two major classes on the basis of the relative lengths of time they take to bring about a 
change in the velocity of a particular enzyme. These are "coarse" and "fine" metabolic control.

\section{Coarse Metabolic Control}

Coarse metabolic control is a long-term (hours to days in eukaryotes; perhaps minutes to hours in rapidly growing prokaryotes), energetically expensive, response that is achieved through changes in the total cellular population of enzyme molecules. The total amount of a given enzyme is dependent upon the relative rates of its biosynthesis versus degradation. Thus, any alteration in the rates of gene expression [i.e., transcription, translation, messenger ribonucleic acid (mRNA) processing or degradation] or proteolysis can be considered as coarse metabolic control. Coarse control can be applied to one or all the enzymes in a particular pathway and most frequently comes into play in response to hormonal stimulation and/ or during tissue differentiation or long-term environmental (adaptive) changes. The dynamic range of coarse metabolic control can be large, particularly when a previously absent enzyme is induced and rises to high levels in response to a stimulus. Coarse metabolic control might appear to be an inefficient and wasteful use of energy since each peptide bond formed requires the hydrolysis of several ATP equivalents, whereas protease activity is not coupled to the production of ATP (but as discussed below can also be ATP-dependent). However, coarse control may be particularly important when a cell must acclimate to changes in its environment, or it becomes necessary to remove abnormal enzyme molecules that have become damaged or arisen by errors in gene expression. In general, the longer the life of an individual cell the more important is the process of intracellular enzyme turnover. For example, in E. coli growing under optimal conditions, mitosis may occur every $20 \mathrm{~min}$. Acclimation of E. coli to its environment occurs largely by the induction or repression of enzyme synthesis. For example, if lactose is added to the growth medium, rapid induction of $\beta$-galactosidase (needed to catabolize lactose) occurs. If lactose is then removed from the medium, the enzyme is not synthesized, and existing $\beta$-galactosidase molecules will be rapidly diluted out within the cytoplasm during the rapid division of cells. In contrast to rapidly dividing microbes, the average lifetime of a cell in a multicellular eukaryote may be several hundred days or more, but many enzymes need to be completely replaced every few days.

Gene Expression The regulation of transcription and translation is covered in Chapters 6 and 7 and will not be discussed in detail here. However, in the context of metabolic control it is important to note that an underlying assumption of many genomic studies is that the expression of a gene at the mRNA level is a quantitative indicator of function of the encoded enzyme. Thus, an $n$-fold increase in transcript levels (detected via Northern blots or gene chip screening) equates to $n$-fold more enzyme and hence $n$-fold more activity. However, it is becoming apparent that this assumption does not always reflect reality. For example, a study that determined fluxes through steps of a central metabolic pathway (glycolysis) in three parasitic protists found that these did not correlate proportionally with the concentration of the corresponding enzymes; that is, relative to various fine metabolic control mechanisms, gene expression alone exerts little control on glycolytic flux.

Protein Turnover Relative to gene expression, much less is known about the mechanisms governing protein degradation. Animal and plant enzymes that coexist in the same cellular compartment may exhibit vastly different turnover rates, ranging from several minutes to hundreds of hours. In general, larger, oligomeric proteins that display complex biological properties and significant hydrophobicity tend to show much shorter half-lives in vivo than do less complex monomeric (and/or less hydrophobic) proteins. It is clear that proteolysis of enzymes can be selectively targeted and may be initiated in response to specific stimuli.

How are Enzymes Selected for Intracellular Degradation? Many enzymes need to first become "tagged" before becoming susceptible to degradation by endogenous proteases. The types of covalent modification used for tagging enzymes for degradation include the formation of a peptide bond between the target enzyme and a protein called ubiquitin, or the modification of the protein by phosphorylation or by oxidation. Ubiquitin $\left(M_{r}\right.$ 9000) is socalled because of its widespread occurrence in eukaryotic cells. Its role in protein turnover has been well-established in animals and plants. Certain proteins destined for degradation are covalently bonded to ubiquitin via the $\mathrm{NH}_{2}$ groups of lysine residues. A single protein may become tagged with many ubiquitin molecules. ATP is required for the ubiquitin conjugation, together with several enzymes. Another method of tagging an enzyme for protease degradation is by phosphorylation, which again is dependent upon the hydrolysis of ATP, in this case by the modifying protein kinase (see below). The ATP requirement of the ubiquitin and phosphorylation tagging systems reflects the bioenergetic cost for endowing the cell with proteolytic specificity. Although tagging methods appear to make many enzymes susceptible to proteolytic attack in vivo, it is not yet clear precisely which features of the target enzymes are recognized by the tagging machinery. 


\section{Fine Metabolic Control}

Fine metabolic controls are generally fast (i.e., seconds to minutes), energetically inexpensive, regulatory devices that modulate the activity of the preexisting enzyme molecule. Operating primarily on the regulatory or pacemaker enzyme(s) of a pathway, fine controls allow the cell to prevent metabolic chaos. Fine controls can be thought of as metabolic transducers that "sense" the momentary metabolic needs of the cell and modulate flux through the various pathways accordingly. It is important to note that the fine metabolic controls discussed in detail below are not mutually exclusive but often interact with, or may actually be dependent upon, one another.

Fine Control 1. Alteration in Substrate Concentration The rate of an enzyme-catalyzed reaction is dependent upon [S] when [S] is subsaturating. Substrate concentrations for most enzymes are subsaturating in vivo. Often the in vivo $[\mathrm{S}]$ is less than or nearly equal to the $K_{m}$ or $S_{0.5}$ value of the enzyme for that substrate. The main exception to this are enzymes such as nucleases, proteases, lipases, phosphorylases, or amylases that catalyze initial steps in macromolecule catabolism, cases where substrate reserves (i.e., glycogen, triglycerides deposits) are huge.

Can pathway flux be controlled by alterations in [S] for any of the enzymes that comprise the pathway? Following stimulation of a metabolic pathway, the concentration of its constituent metabolites may increase severalfold (frequently 2- to 5-fold). However, pathway flux may increase by as much as 100 -fold under the same stimulation. For enzymes that obey hyperbolic substrate saturation kinetics (i.e., Michaelis-Menten kinetics where Hill coefficient or $n_{H}=1$ ) (Fig. 1.1a), Table 1.2 shows that the increase in [S] must be about 80-fold in order to obtain a 9-fold change in the activity of the enzyme. Such an increase in

TABLE 1.2 Quantitative Influence of Hyperbolic versus Sigmoidal Substrate $(S)$ Saturation Kinetics on an Enzyme's Response to Variations in Its Substrate Concentration ${ }^{a}$

\begin{tabular}{ll}
\hline & $\begin{array}{c}\text { Required Change in [S] to Increase } V_{0} \text { from } \\
10 \text { to } 90 \% \text { of } V_{\max }\end{array}$ \\
\hline 0.5 & 6561 -fold \\
1.0 & 81 -fold \\
2.0 & 9 -fold \\
3.0 & 4.33 -fold \\
4.0 & 3 -fold \\
\hline
\end{tabular}

${ }^{a}$ The parameter $n_{H}$ represents the Hill coefficient, derived by fitting enzyme initial velocity $\left(V_{0}\right)$ versus $[\mathrm{S}]$ data to the Hill equation.

Source: Adapted from Price and Stevens (1989).
$[\mathrm{S}]$ is rarely if ever seen and so variation in $[\mathrm{S}]$ cannot be the sole determinant of the in vivo activity of enzymes that follow hyperbolic saturation kinetics.

Not all enzymes show simple Michaelis-Menten kinetics, however. Multimeric enzymes (i.e., consisting of two or more polypeptides in their native state) often contain more than one substrate binding site. Binding of substrate to one subunit can affect the conformation of other subunits and positively enhance the binding of substrate to them. The result of such cooperative binding of substrate is a sigmoidal relationship between enzyme activity and [S] (Fig. 1.1b). Enzymes of this nature have been termed allosteric because they can assume "other shapes" or conformations by the reversible, noncovalent binding of a specific metabolite. Sigmoidal substrate saturation kinetics has been referred to as homotropic allosterism since the allosteric modulator and the substrate are identical. Table 1.2 shows that the same 9-fold increase in activity that required an 80 -fold increase in substrate concentration for a hyperbolic enzyme can be achieved with only a 3-to 4-fold increase in [S] for a sigmoidal enzyme (Table 1.2). The actual increase in [S] that is required is dependent upon the degree of cooperativity with which the enzyme binds its substrate. With increased cooperativity (i.e., higher values for $n_{H}$ reflected by increasing sigmoidal $V$ vs. [S] plots) smaller fold increases in [S] are required to give the same relative increase in enzyme activity.

In summary, changes in substrate concentrations that normally occur in vivo can alter the rate of pathway flux but do so most effectively for enzymes that show sigmoidal saturation kinetics (i.e., homotropic allosterism). Enzymes of this nature have been found in all phyla. Invariably such enzymes have been identified as pacemakers. Note, however, that not all multimeric or pacemaker enzymes necessarily display sigmoidal substrate saturation kinetics. Although sigmoidal kinetics allow a much more sensitive control of reaction rate by [S], it should be stressed that an alteration in [S] as a mechanism of fine control is often unimportant, relative to the other fine controls discussed below. The real metabolic "advantage" of sigmoidal substrate saturation kinetics is that this may allow metabolite activators (and/or covalent modification) to facilitate a marked enhancement in enzyme activity (at a relatively constant $[\mathrm{S}]$ ) by promoting an allosteric transition that brings about a reversible shift between hyperbolic and sigmoidal saturation kinetics (see below).

Fine Control 2. Variation in $\mathbf{p H}$ Most enzymes have a characteristic $\mathrm{pH}$ at which their activity is maximal, that is, the $\mathrm{pH}$ optimum. Above or below this $\mathrm{pH}$ the activity normally declines, although to varying degrees depending upon the particular enzyme. Thus, enzymes can show $\mathrm{pH}$ versus activity profiles ranging in shape from very broad 


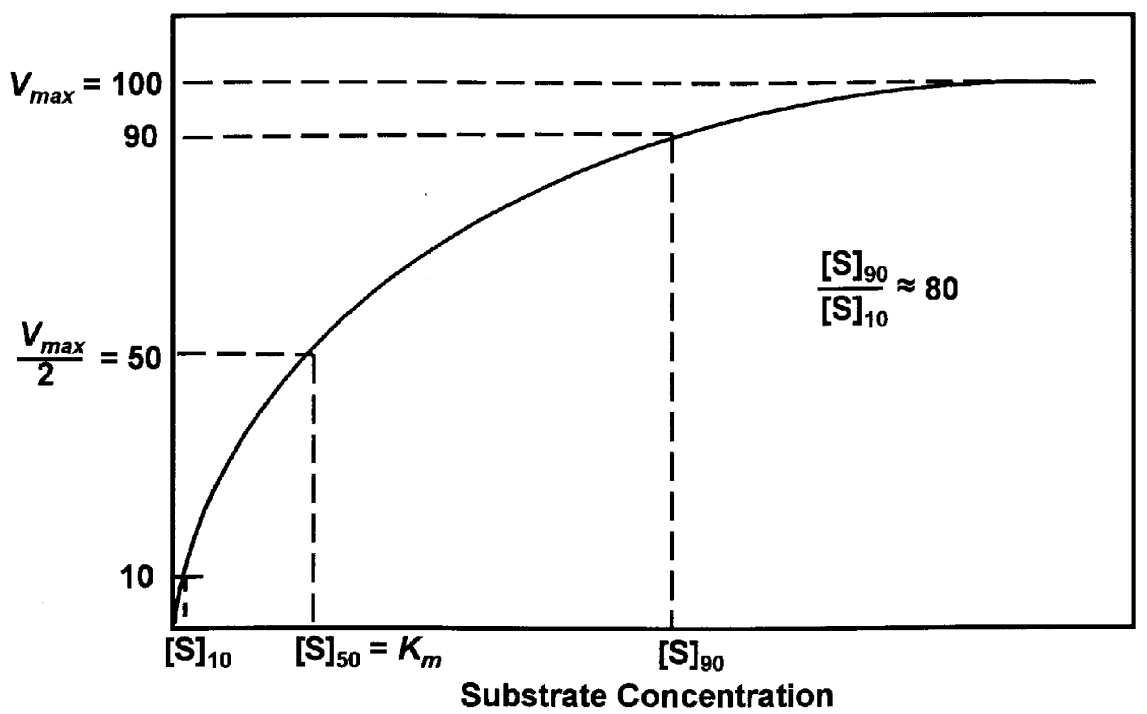

(a)

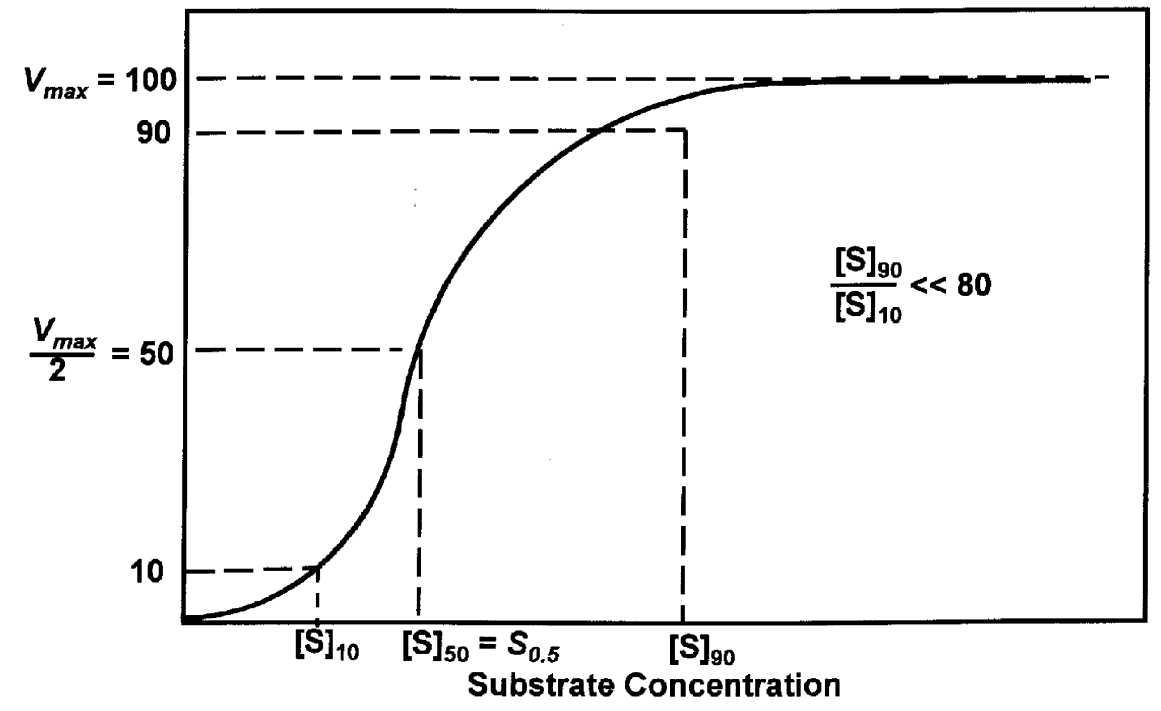

(b)

Figure 1.1 Relationship between substrate concentration and reaction rate for enzymes that follow (a) hyperbolic or $(b)$ sigmoidal substrate saturation kinetics. $[\mathrm{S}]_{90},[\mathrm{~S}]_{50}$, and $[\mathrm{S}]_{10}$ denote the respective substrate concentration that yields 90,50 , and $10 \%$ of $V_{\max }$, respectively. To increase the activity of an enzyme that follows hyperbolic substrate saturation kinetics $(a)$ from 10 to $90 \%$ of $V_{\max }$, the increase in its [S] must be about 80 -fold. A much lower increase in [S] is needed to achieve the same relative increase in the activity of an enzyme that follows sigmoidal substrate saturation kinetics (see text and Table 1.2 for details).

to very narrow. As the $\mathrm{pH}$ optimum of an enzyme is not always the same as the $\mathrm{pH}$ of its intracellular surroundings, this suggests that the $\mathrm{pH}$ dependence of enzyme activity may be one factor that determines its overall activity in the cell. As all cells contain thousands of enzymes, many of which show very different responses to $\mathrm{pH}$, the intra- cellular $\mathrm{pH}\left(\mathrm{pH}_{\mathrm{i}}\right)$ may represent an important element of fine metabolic control.

The light-dependent activation of several of the enzymes of the reductive pentose-phosphate pathway (CalvinBenson cycle) provides a well-documented example of how changes in $\mathrm{pH}_{\mathrm{i}}$ can contribute to metabolic control 


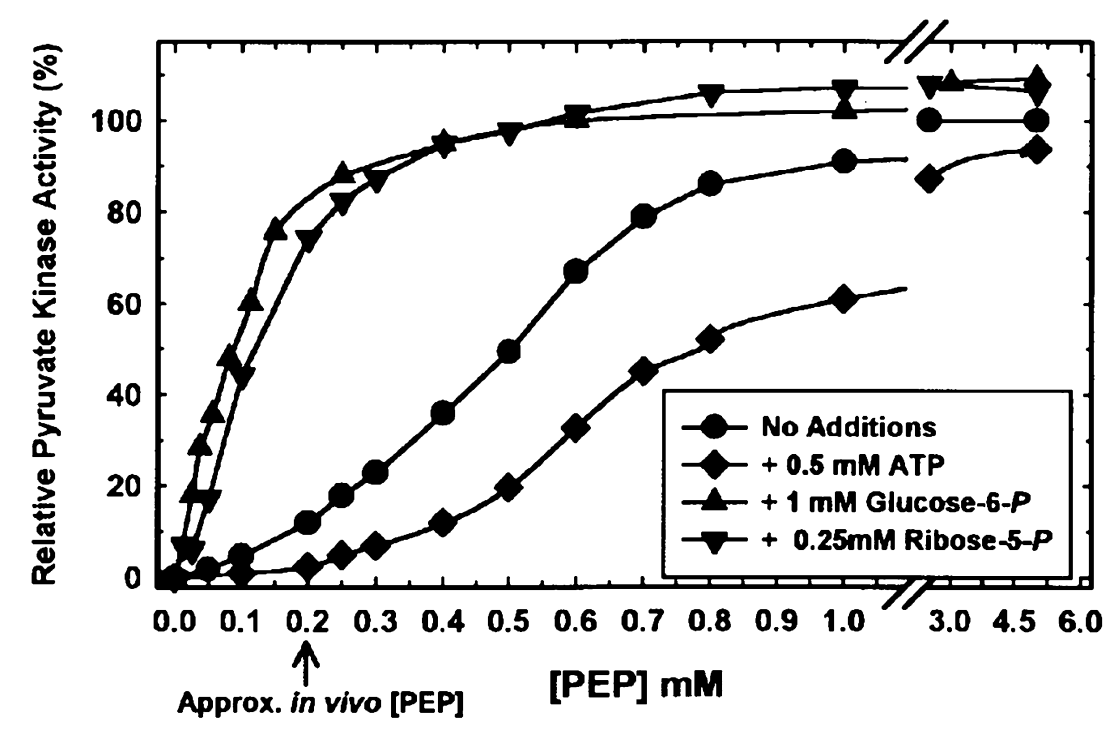

Figure 1.2 Influence of metabolite effectors on the substrate saturation kinetics of purified pyruvate kinase from the cyanobacterium Synechoccous PCC 6301 (adapted from Knowles et al., 2001). Note that at the approximate concentration of PEP (phosphoenolpyruvate) substrate that occurs in vivo $(\sim 0.2 \mathrm{mM})$, the addition of physiological concentrations of glucose-6-phosphate or ribose-5phosphate can raise pyruvate kinase activity from $\sim 10 \%$ of $V_{\max }$ to $\sim 90 \%$ of $V_{\max }$, whereas the inhibitor (ATP) can suppress activity to $<2 \%$ of $V_{\max }$.

within the chloroplast stroma of plants. Photosynthetic electron transport has been linked to $\mathrm{H}^{+}$uptake from the stroma into the thylakoid lumen. This establishes a proton gradient between the lumen and stroma that drives the photophosphorylation of ADP by $\mathrm{P}_{\mathrm{i}}$ (inorganic phosphate) to form ATP. This ATP is used to help power the biosynthetic $\left(\mathrm{CO}_{2}\right.$ fixation) reactions in the stroma. The transport of $\mathrm{H}^{+}$ions into the lumen results in a light-dependent increase in stromal $\mathrm{pH}_{\mathrm{i}}$ from about 7.0 to 8.0 , and the high $\mathrm{pH}$ facilitates $\mathrm{CO}_{2}$ fixation because several enzymes of the reductive pentose-P pathway have relatively sharp alkaline $\mathrm{pH}$ optima of between 7.8 and 8 . In the dark, however, $\mathrm{H}^{+}$ions leak back into the stroma and stromal $\mathrm{pH}_{\mathrm{i}}$ falls back to 7.0. Both the drop in stromal $\mathrm{pH}$ and the limitation of ATP supply in the dark combine to help suppress the reductive pentose-P pathway until the reintroduction of light makes the system fully operational once again.

Fine Control 3. Allosteric Effectors Multisubunit regulatory enzymes often contain allosteric sites that are separate from the active or catalytic site where specific inhibitor or activator metabolites can bind reversibly. By varying the concentration of these nonsubstrate effector molecules, it is possible to markedly alter the activity of an enzyme and thereby modify the flux of metabolites through an entire pathway. Allosteric effectors alter enzyme activity by binding to an allosteric site and eliciting a precise change in the enzyme's conformation. This conformational change has been termed heterotropic allosterism because, in contrast to homotropic allosterism, the allosteric modulator is a molecule other than the substrate. The amount of an allosteric modulator bound to the corresponding allosteric site of an enzyme is a reflection of the concentration of allosteric modulator and the enzyme's affinity for this particular ligand. Allosteric effectors themselves may show hyperbolic or sigmoidal (i.e., cooperative) saturation kinetics. As outlined above for sigmoidal substrate saturation kinetics, a sigmoidal relationship would allow a very sensitive response of enzyme conformation (and hence activity) to changes in effector concentration. The conformational change brought about by the binding of an allosteric effector thereby promotes (activators) or hinders (inhibitors) enzyme-substrate interactions such that some or all of the kinetic constants may be significantly altered including $V_{\max }, K_{m}$ or $S_{0.5}$, and $n_{H}$. This regulatory strategy allows metabolites that are remote from a specific reaction to function as feedforward (activators) or feedback (inhibitors) control signals on enzyme activity. The key significance of this interaction is that, at the low and relatively constant substrate concentrations that usually exist in vivo, the rate of a reaction can be dramatically increased or decreased by effector binding. This is illustrated graphically by the example illustrated in Figure 1.2 that shows that by addition of an activator or inhibitor it is possible to vary the rate of an allosteric 
enzyme reaction from less than $10 \%$ to nearly $100 \%$ of $V_{\max }$ with little or no change in [S].

Essentially all pacemaker enzymes are at least partially controlled by allosteric effectors. As indicated above, for an effector to have meaningful regulatory significance, the concentration at which it significantly activates or inhibits in vitro must be close to the effector's actual concentration range in vivo.

Enzyme Activation Activators are metabolites that reversibly interact at an allosteric activator site. When bound, they elicit an allosteric transition that typically elevates the reaction rate at subsaturating $[\mathrm{S}]$ by causing a reduction in the enzyme's $K_{m}$ (or $S_{0.5}$ ) value. This may or may not be accompanied by a corresponding increase in $V_{\max }$. This is illustrated by the example given in Figure 1.2, which concerns purified pyruvate kinase from the cyanobacterium, Synechococcus. Pyruvate kinase is an important control enzyme of glycolysis in all phyla and catalyzes the essentially irreversible reaction: phosphoenolpyruvate (PEP) + ADP $\rightarrow$ pyruvate + ATP. The cyanobacterial pyruvate kinase was fully purified and shown to be potently activated by glucose-6-phosphate and ribose-5-phosphate, the immediate products of glycogen breakdown. Low concentrations of either activator caused a slight increase in enzyme $V_{\max }$ but elicited a marked enhancement in PEP binding, which was accompanied by a shift from sigmoidal to hyperbolic PEP saturation kinetics (Fig. 1.2). The concentrations of glucose-6-P and ribose-5-P required for half-maximal activation (i.e., $K_{a}$ values) were less than $25 \mu \mathrm{M}$, which suggests that the activating effects observed in vitro are relevant to pyruvate kinase (PK) and glycolytic flux control in vivo.

Enzyme Inhibition Any substance that reduces the rate of an enzyme-catalyzed reaction is called an inhibitor. Competitive (or specific) inhibition is the most common form of reversible enzyme inhibition and arises when the inhibitor and substrate compete for a common substrate binding site (i.e., the active site) so that when one binds the other cannot. The effect of a competitive inhibitor is to decrease the apparent affinity of the enzyme for its substrate without any effect on the reactivity of the enzyme-substrate complex once formed. Thus, competitive inhibition causes an increase in the $K_{m}$ (or $S_{0.5}$ ) value, does not affect $V_{\max }$, and is overridden by increasing the [S]. Although competitive inhibitors are not allosteric inhibitors (because they do not bind to an allosteric site, a site that is distinct from the active site), they nevertheless constitute an important aspect of enzyme control in vivo. For example, potent competitive inhibition of many microbial, yeast, and plant acid phosphatases by their product, inorganic phosphate $\left(\mathrm{P}_{\mathrm{i}}\right)$, is in accord with the hypothesis that acid phosphatases are particularly active in vivo during nutri- tional $\mathrm{P}_{\mathrm{i}}$ starvation when intracellular $\mathrm{P}_{\mathrm{i}}$ levels become depleted. Conversely, any accumulation of cellular $P_{i}$ due to $\mathrm{P}_{\mathrm{i}}$ resupply would act as a tight regulatory control to prohibit further hydrolysis of certain phosphorylated compounds by the various intracellular acid phosphatases.

Mixed-type inhibition is a rarer, but important, form of metabolic control in which the inhibitor reversibly interacts with the enzyme, or enzyme-substrate complex, at a true allosteric site. In this instance $V_{\max }$ is reduced, whereas $K_{m}$ is increased. Mixed inhibition is frequently misconstrued as noncompetitive inhibition, which theoretically reduces an enzyme's catalytic potential (i.e., $V_{\max }$ ) without affecting substrate binding (i.e., $K_{m}$ or $S_{0.5}$ ). However, it is unlikely for an inhibitor to interact with an enzyme and alter its $V_{\max }$ without having any effect on substrate binding. Moreover, most commonly quoted examples of noncompetitive inhibition prove, on reexamination of the original data, to be examples of mixed inhibition.

Interacting Effectors The activity of an allosteric enzyme in vivo is dependent upon the relative concentrations of its activators versus inhibitors. Often, the presence of an activator can override, or cancel, inhibitory signals. Thus, it is the ratio of [activator] : [inhibitor], rather than their absolute concentrations per se, that is believed to be a major factor in determining the overall activity of an allosteric enzyme in vivo.

Adenine Nucleotides as Metabolic Effectors Energy transduction and energy storage, involving the adenine nucleotides (ATP, ADP, and AMP), are fundamental features of metabolism in all organisms. Thus, it is not surprising that the adenine nucleotides (particularly ATP and AMP) are potent allosteric effectors of many enzymes, their effects having a major impact on the balance between ATP formation by catabolic pathways and ATP use by many other cell functions. In most tissues, the adenine nucleotides are maintained in equilibrium by the enzyme adenylate kinase (also known as myokinase in muscles), which catalyzes the interconversion of the three adenine nucleotides: ATP + AMP $\longleftrightarrow 2$ ADP. Since the concentration of adenine nucleotides in a healthy cell are $[\mathrm{ATP}]>[\mathrm{ADP}] \gg[\mathrm{AMP}]$, and the reaction catalyzed by adenylate kinase is very close to equilibrium in vivo, any small change in the balance between ATP and ADP will be transmitted (by adenylate kinase) into a relatively large change in [AMP]. Thus, enzymes that are allosterically regulated by AMP can respond with high sensitivity to small changes in [ATP] and [ADP]. For example, in insect flight muscle, [ATP] falls by about $10 \%$ during the early seconds of flight, and this causes an immediate $250 \%$ increase in [AMP]. AMP is a potent allosteric activator of key control enzymes such as PFK, and via allosteric effects as well as the action of the AMP- 
activated protein kinase (see below), AMP functions as an "amplifier" of relatively small changes in ATP concentration. Although not sufficient to fully explain the approximately 100-fold increase in glycolytic flux during insect flight, it is believed that the increase in [AMP] makes a major contribution to the response.

The widespread importance of adenine nucleotides in metabolic regulation led Atkinson in 1977 to introduce the concept of the adenylate energy charge (AEC), which is defined as

$$
\mathrm{AEC}=\frac{[\mathrm{ATP}]+\frac{1}{2}[\mathrm{ADP}]}{[\mathrm{ATP}]+[\mathrm{ADP}]+[\mathrm{AMP}]}
$$

The AEC can vary theoretically between 0 (i.e., all adenylates present as AMP) and 1 (i.e., all adenylates present as ATP), but in healthy cells AEC is normally controlled at a value of between 0.7 and 0.9 . Fluctuations in adenylate levels under different circumstances provide a basis for metabolic control by the AEC. Regulatory enzymes that occur in pathways in which ATP is consumed (i.e., anabolic pathways) respond to changes in AEC (via the action of ATP as an activator and/or AMP as an inhibitor) in the general way shown by curve 2 of Figure 1.3. Regulatory enzymes such as PFK that occur in pathways in which ATP is generated (i.e., catabolic pathways) respond to changes in AEC (via the action of ATP as an inhibitor and/or AMP as an activator) in the general way shown by curve 1 of Figure 1.3. It is clear from Figure 1.3 that any fall in AEC would be counteracted by a consequent increase in flux through ATP-generating pathways (curve 1 ) and by a decrease in flux through ATP-consuming pathways (curve 2).

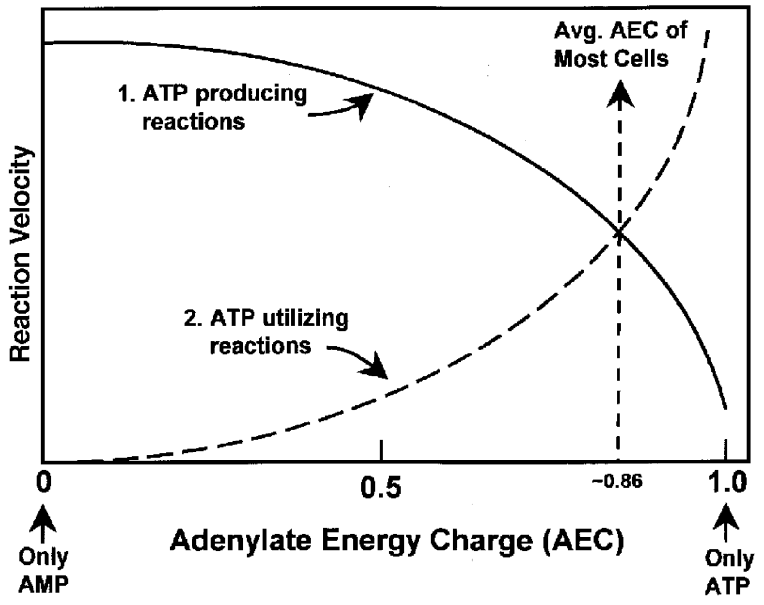

Figure 1.3 Responses to adenylate energy charge by regulatory enzymes in metabolic pathways that produce ATP (curve 1) or consume ATP (curve 2) (adapted from Atkinson, 1977).
Fine Control 4. Reversible Covalent Modification A wide variety of posttranslational modifications of proteins can have an influence on enzymatic activity. Some of these, such as the ubiquitin-tagging system mentioned above, are irreversible and are not mechanisms of fine metabolic control. Similarly, proteolytic cleavage of an inactive precursor protein (or zymogen) is not a mechanism of fine control, although it is an important mechanism for safely synthesizing and storing various enzymes (e.g., digestive enzymes) until they are released at their site of action (e.g., in the lysosome of animal cells).

Reversible covalent modification (also known as an interconvertible enzyme cascade) plays a dominant role in fine metabolic control. The general model is that an enzyme is interconverted between a less active (or inactive) and more active form because of the effects of the covalent modification on its substrate saturation kinetics and/or response to allosteric effectors (Fig. 1.4a). This interconversion is not the result of a reversible equilibrium, as is the case for regulation by allosteric effectors (Fig. 1.4b) but is usually governed by two thermodynamically favorable enzyme-catalyzed reactions that result in the formation of new stable covalent bonds on the surface of the target enzyme. Interconversion in either direction can be very fast (e.g., seconds to minutes) and very complete (up to $100 \%$ conversion). A change in enzyme conformation that is induced by covalent modification normally causes an alteration of enzyme-substrate interactions such that kinetic parameters such as $V_{\max }, K_{m}$ (or $S_{0.5}$ ), and $n_{\mathrm{H}}$ are significantly elevated or reduced. The sensitivity of the target enzyme to allosteric activators or inhibitors can also be affected. Covalent modification can quickly provide the cell with an essentially "new" enzyme form whose kinetic properties are geared to the cell's momentary metabolic requirements.

Enzyme control by reversible covalent modification is the major mechanism whereby extracellular signals, such as hormones, nervous impulses, and numerous environmental stimuli, can coordinate the control of key enzymes of intracellular pathways. Reversible covalent modification of enzymes typically allows a very marked sensitivity (e.g., "amplification") to signals, much greater than is possible for an enzyme responding to allosteric effectors, and indeed, often regulates enzymes in a virtual on-off manner (Fig. 1.4).

Although at least 150 types of posttranslational modifications of proteins have been reported in vivo, very few appear to be important in the control of enzyme activity. The major kinds of reversible covalent modifications that reversibly control enzyme activity are summarized in Table 1.3. Protein phosphorylation was the first to be discovered and has emerged as the predominant method of reversible covalent modification used in eukaryotic metabolic control. Although reversible enzyme phosphorylation 


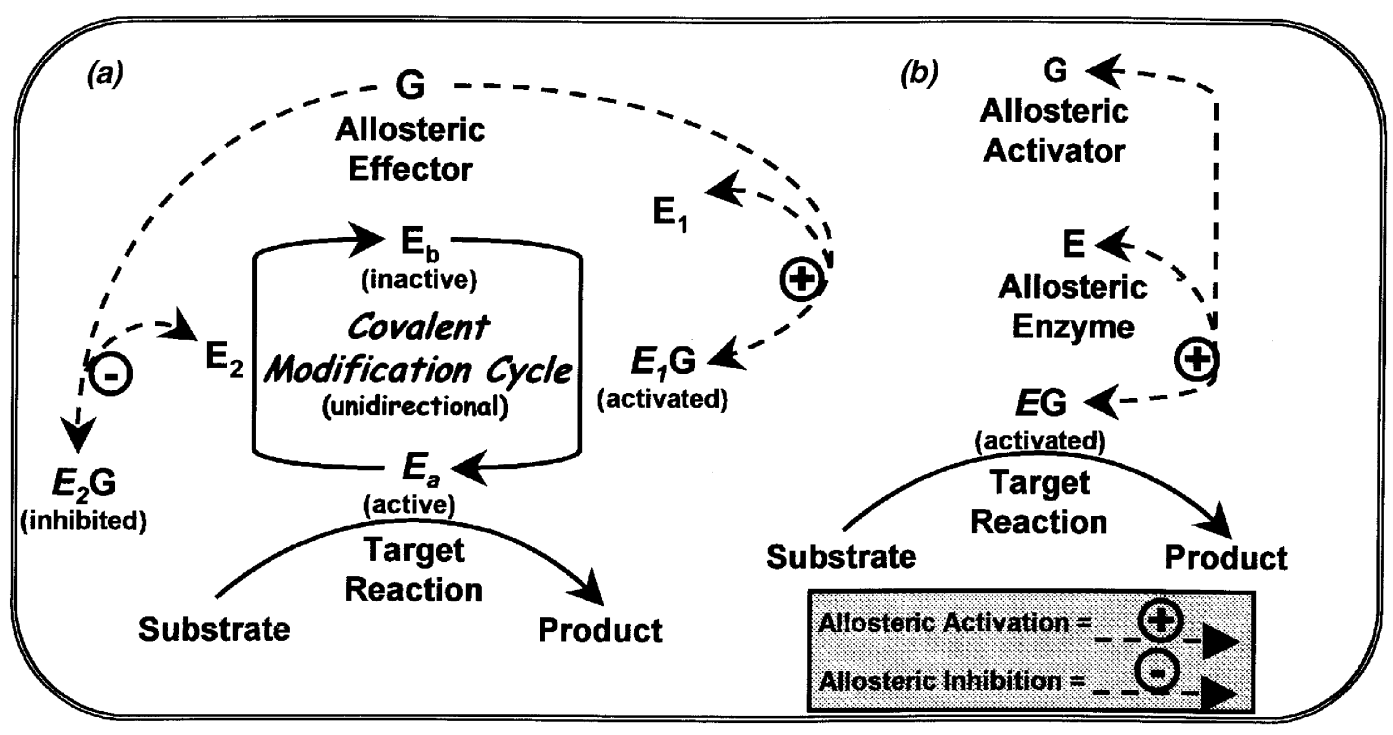

Figure 1.4 General model for enzyme control by reversible covalent modification and allosteric activation $(a)$ In reversible covalent modification an effector $\mathrm{G}$ typically exerts reciprocal allosteric effects on the modifying enzymes $\left(\mathrm{E}_{1}\right.$ and $\left.\mathrm{E}_{2}\right)$ that catalyze the covalent interconversion of a target enzyme between inactive (or less active) and active conformations. Additional substrates (such as ATP and water) are needed to make the respective interconversion reactions thermodynamically irreversible. (b) In conventional allosteric activation the activator $\mathrm{G}$ reversibly binds directly to the enzyme to enhance its activity (adapted from Fell, 1997).

does not appear to play as prominent a role in the control of prokaryotic metabolism, it does play a significant role in the control of some prokaryotic enzymes. Reversible ADP-ribosylation, methylation, and adenylation also contribute to the fine control of various enzymes in eukaryotes and/or microbes. In addition, enzyme dithiol-disulfide interconversion is important in the control of plant metabolism. To further illustrate the principles and critical importance of reversible covalent modification in fine metabolic control, phosphorylation-dephosphorylation, and to a lesser extent dithiol-disulfide interconversion will be discussed.
Phosphorylation-Dephosphorylation Enzyme modification by the reversible covalent incorporation of phosphate is a widespread phenomenon with considerable consequences for metabolic control in vivo. As outlined in Figure $1.5 a$, the protein phosphorylation reaction is catalyzed by a protein kinase that typically transfers the $\gamma$-phosphate of ATP to a hydroxyl group on a serine, threonine, or tyrosine residue of the target protein. The reverse reaction is catalyzed by a phosphoprotein phosphatase. Both protein kinases and phosphoprotein phosphatases may have wide or narrow substrate specificities and are themselves always subject to reciprocal fine metabolic

TABLE 1.3 Major Classes of Reversible Enzyme Covalent Modification Important in Metabolic Control

Type of Covalent

Phosphorylation Disulfide-dithiol interconversion

Nucleotidylation ADP-ribosylation Methylation $\begin{array}{ll}\text { Mainly ATP (also GTP) } & \text { Ser, Thr, Tyr, His } \\ \text { Reduced thioredoxin } & \text { Cys }\end{array}$

ATP, UTP Tyr, Ser

$\mathrm{NAD}^{+} \quad$ Arg, Glu, Lys

$S$-adenosyl-methionine

Asp, Glu, Lys, His, Gln

More widespread in eukaryotes than in prokaryotes Vascular plants and green algae

Only in prokaryotes

Eukaryotes and prokaryotes

Eukaryotes and prokaryotes 


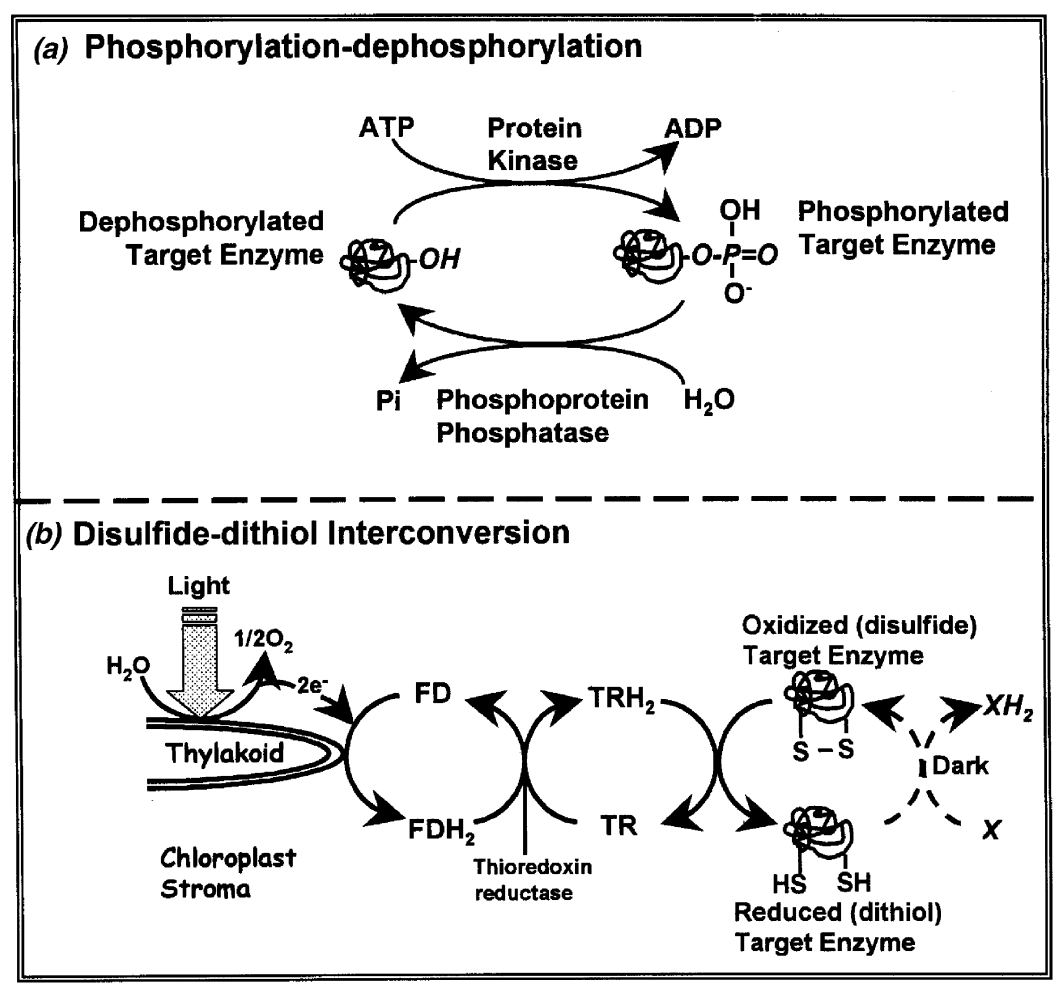

Figure 1.5 Examples of the control of enzyme activity by reversible covalent modification. For details refer to the text. (a) Phosphorylation-dephosphorylation and (b) disulfide-dithiol interconversion. Abbreviations: $\mathrm{FD}$ and $\mathrm{FDH}_{2}$, oxidized and reduced ferredoxin, respectively; $\mathrm{P}_{\mathrm{i}}$, inorganic phosphate; TR and $\mathrm{TRH}_{2}$, oxidized and reduced forms of thioredoxin, respectively; $\mathrm{X}$, some oxidant normally kept reduced in the light.

controls. To prevent a futile cycle that wastes ATP, under conditions where a protein kinase is active, the corresponding phosphatase is usually inhibited and vice versa. In animals, plants, and yeast there are significant similarities in amino acid sequences among most of the known protein kinases and phosphoprotein phosphatases indicating that the interconverting enzymes belong to extended families of proteins that have probably evolved from common ancestors.

Since more than $10 \%$ of the 10,000 proteins in a normal mammalian cell are thought to be controlled by reversible phosphorylation, this aspect of metabolic control has received considerable attention. Abnormal protein phosphorylation is the basis for or the result of major human diseases including cancer, diabetes, and arthritis. Mutations of certain protein kinases and phosphatases underlie a number of hereditary diseases, including certain leukemias. Many drugs are specific inhibitors of protein kinases and phosphatases. For example, by potently inhibiting of a specific type of phosphoprotein phosphatase, the immunosuppressant drug cyclosporin plays a key role in the success of organ transplants. Likewise, it is probable that protein kinases and/or phosphatases will become critical targets for metabolic engineering.
The first example of enzyme control by reversible covalent modification was provided in the mid-1950s by Edwin Krebs and Edmond Fischer who were studying the control of muscle glycogen phosphorylase, the enzyme that cleaves glucose-1-phosphate units from the glycogen polymer. Phosphorylase $b$, the less active enzyme form, was found to be converted to the more active phosphorylase $a$ by the covalent attachment of a phosphate group, derived from ATP, to a serine residue on the protein, catalyzed by a protein kinase that was named phosphorylase kinase. Conversely, a phosphatase was discovered that catalyzed the dephosphorylation and conversion of phosphorylase $a$ back to the less active phosphorylase $b$. This seminal work led to the subsequent discovery by Earl Sutherland of the hormone-mediated production of cyclic $3^{\prime}, 5^{\prime}$-adenosine monophosphate (cAMP) by the enzyme adenylyl cyclase and, thus, the concept of the second messenger as an intracellular transducer of extracellular (i.e., hormonal) signals. It has since become clear that there are many other signals and second messengers apart from hormones and cAMP that influence protein phosphorylation status (Table 1.4). To complicate matters even further, although protein phosphorylation may lead to a change in enzyme 
TABLE 1.4 Classification of Major Serine and Threonine Protein Kinases Important in Metabolic Control

\begin{tabular}{lll}
\hline Signal & \multicolumn{1}{c}{ Protein Kinase Class } & \multicolumn{1}{c}{ Distribution } \\
\hline Cyclic nucleotides & cAMP-dependent protein kinase & Animals and yeast, (plants?) \\
$\mathrm{Ca}^{2+} /$ calmodulin & cGMP-dependent protein kinase & \\
$\mathrm{Ca}^{2+}$ & $\mathrm{Ca}^{2+} /$ calmodulin protein kinases & Animals and yeast \\
Diacylglycerol $/ \mathrm{Ca}^{2+}$ & $\mathrm{Ca}^{2+}$-dependent protein kinase & Plants \\
AMP & Protein kinase C & Animals and plants (yeast?) \\
Metabolic intermediates & AMP-dependent protein kinase & Animals and yeast (plants?) \\
\hline
\end{tabular}

activity, many enzymes are subject to multiple phosphorylations at different sites, and some of these phosphorylations appear to affect the ability of protein kinases and phosphatases to phosphorylate or dephosphorylate other sites on the same target enzyme.

Protein Kinases The central role of phosphorylationdephosphorylation in cell biology and metabolic control is illustrated by the fact that the largest known protein family consists of the eukaryotic protein kinase superfamily. These enzymes are related by virtue of their similar kinase domains, which consist of about 250 to 300 amino acid residues. The rapid pace of discovery of members of the eukaryotic protein kinase superfamily can be attributed to the development of molecular cloning and genome sequencing technologies, as well as the advent of the polymerase chain reaction, which stimulated the use of similarity-based cloning strategies. It has been estimated that the mammalian and yeast genomes contain approximately 1000 and 120 different protein kinase genes, respectively, comprising 1 to $2 \%$ of all genes! Similarly, in the vascular plant Arabidopsis thaliana there are believed to be as many as 2500 different protein kinase genes (comprising more than $5 \%$ of its entire genome). Identifying the mechanisms that control the activity of each protein kinase, as well as the endogenous protein substrates, for the myriad of eukaryotic protein kinases and the influence of phosphorylation on their biological activities will remain a daunting task for many years to come. Apart from mammals and vascular plants, eukaryotic protein kinase superfamily members have been found in a wide range of other animal phyla, as well as fungi and protists. Tyrosine protein kinases appear to be associated with the receptors for certain hormones such as insulin, and although undoubtedly important, they do not appear to play as direct a role in the fine control of metabolic enzymes as do the serine/ threonine protein kinases. As outlined in Table 1.4, each major class of protein kinase is allosterically stimulated by a specific signal metabolite that makes enzyme phosphorylation responsive to extracellular and/or intracellular signals.
Protein Kinases That Respond to Extracellular Signals Relative to direct allosteric control of a target enzyme, protein-kinase-mediated modulation of the activity of the same target enzyme is usually initiated by a much smaller change in allosteric effector concentration and can thus provide a much larger amplification of the initial signal. This is illustrated by the aforementioned regulation of glycogen phosphorylase, in which the conversion of the inactive $b$ form to the active $a$ form is catalyzed by phosphorylase kinase, which is, in turn, controlled by reversible phosphorylation. As outlined in Figure 1.6, a sequence of events can be traced back to the binding of a hormone (i.e., epinephrine in muscle or glucagon in liver) to a receptor on the plasma membrane. Hormone binding stimulates the synthesis of the second messenger, cAMP, and it has been demonstrated that there is only a very small increase (i.e., $<1 \mu \mathrm{M}$ ) in the concentration of cAMP upon treatment of muscle tissue with a hormone such as epinephrine. However, each molecule of cAMP-dependent protein kinase (also known as protein kinase A) that is activated can itself rapidly activate (phosphorylate) many molecules of phosphorylase kinase, which then activates (phosphorylates) an even larger number of glycogen phosphorylase molecules, and leads to a rapid increase in glycogen breakdown. It has been estimated that $50 \%$ of glycogen phosphorylase $b$ could be converted to the active $a$ form with only a $1 \%$ increase in the concentration of cAMP, the process occurring within about $2 \mathrm{~s}$ after hormone administration.

The same phosphorylation mechanism that activates glycogen phosphorylase also inactivates the key pacemaker enzyme of glycogen synthesis, glycogen synthetase, and vice versa (Fig. 1.7). This precludes futile cycling between glycogen and glucose-1-phosphate and illustrates the crucial point that when antagonistic (oppositely directed) enzymes in potential futile cycles are phosphorylated, it is invariable that one is activated whereas the other is inhibited. Although there are exceptions to the rule, in general it appears that the activities of catabolic and anabolic enzymes (such as glycogen phosphorylase and glycogen synthetase), which are subject to this mechanism of 


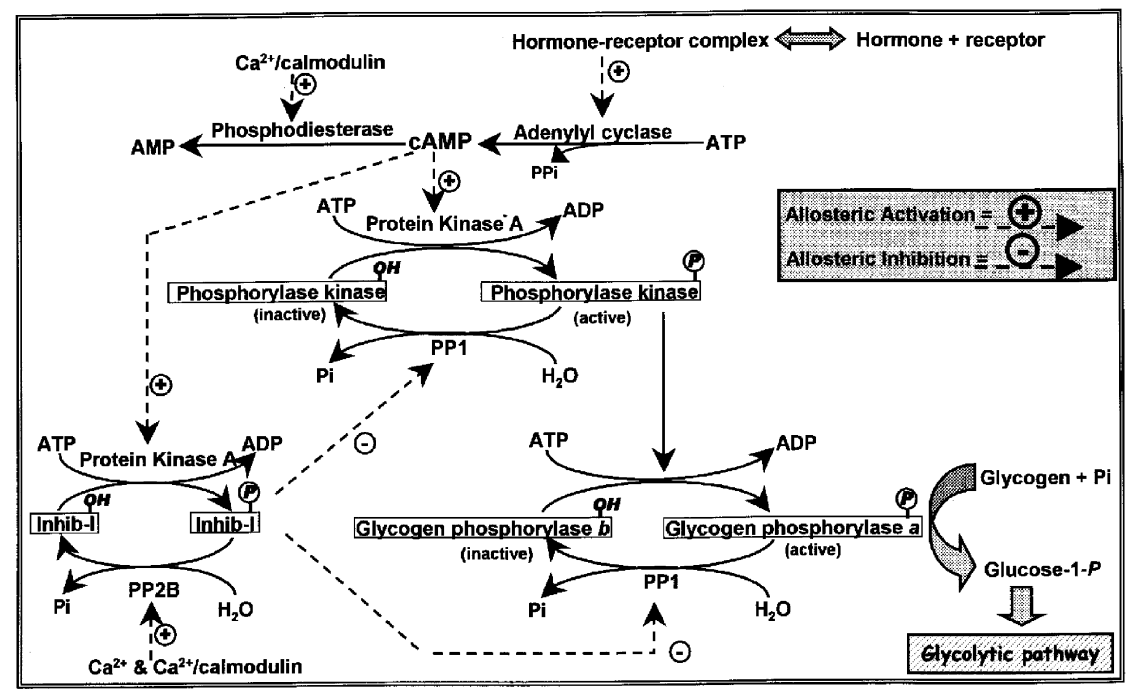

Figure 1.6 The glycogen phosphorylase activation cascade in mammalian muscle. Abbreviations: Inhib-1, Inhibitor 1; PP1 and PP2B, protein phosphatase 1 and 2B, respectively (adapted from Fell, 1997).

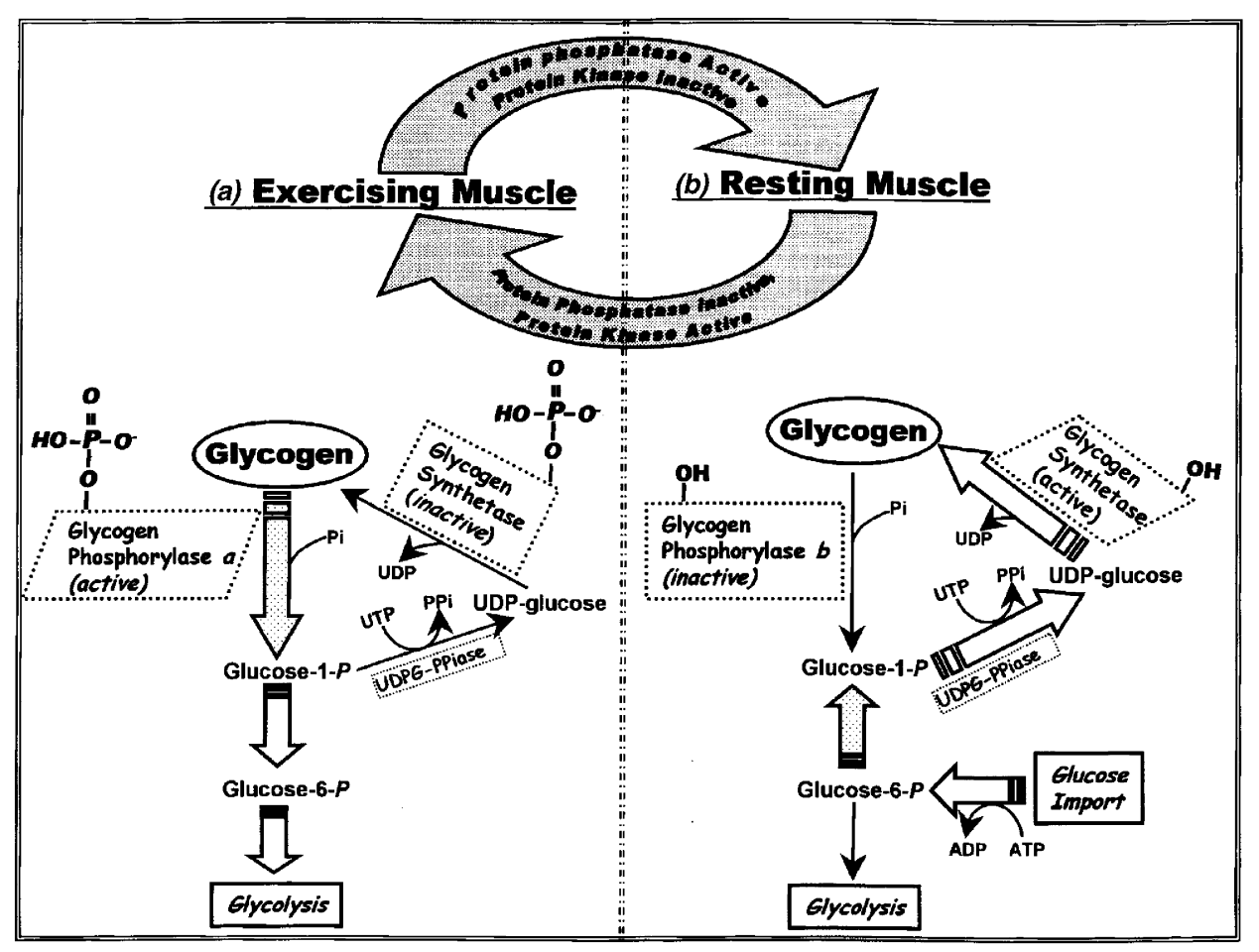

Figure 1.7 Reciprocal control of glycogen phosphorylase and glycogen synthetase by proteinkinase-mediated phosphorylation prevents futile cycling between glycogen and glucose-1-phosphate. (a) In exercising muscle hormonal or neuronal signals lead to second-messenger-mediated (i.e., cAMP or $\mathrm{Ca}^{2+}$ ) protein kinase activation and the consequent phosphorylation/activation of glycogen phosphorylase and phosphorylation/deactivation of glycogen synthetase. (b) In resting muscle the corresponding protein phosphatase is activated to dephosphorylate both enzymes leading to inactivation and activation of glycogen phosphorylase and glycogen synthetase, respectively. Abbreviations: PPi, pyrophosphate; UDPG-PPase, UDP-glucose pyrophosphorylase. 
fine control, are, respectively, activated and inhibited in their phosphorylated form.

Low cAMP concentrations are maintained via the action of a variety of phosphodiesterase isozymes that convert cAMP to AMP and are generally activated by $\mathrm{Ca}^{2+} /$ calmodulin (see Fig. 1.6 and below). cAMP is a true signal metabolite whose only role is to control enzyme activities; it is not a metabolic intermediate for the synthesis of any other compound. Various diseases and pathological states in humans arise through "incorrect" protein phosphorylations caused by either abnormally high or low intracellular levels of cyclic AMP (see Text Box 1.2). In contrast to animal systems, cAMP has no confirmed effect on the phosphorylation of plant proteins (although plant cells have been reported to contain cAMP).

\section{TEXT BOX 1.2 CYCLIC NUCLEOTIDES-TOO MUCH OR TOO LITTLE IS UNHEALTHY}

Many diseases and pathological states in humans arise from the incorrect phosphorylation of proteins brought about by abnormal levels of cellular second messengers. For example, the cholera toxin causes elevated cAMP levels in intestinal epithelial cells due to its continual stimulation of adenylyl cyclase in these cells (adenylyl cyclase converts ATP into cAMP). The cholera toxininduced elevation in cAMP levels in intestinal epithelial cells leads to all of the symptoms associated with this debilitating disease. By contrast, impotence in human males is associated with abnormally low $3^{\prime}, 5^{\prime}$-cyclic guanosine monophosphate (cGMP) levels in erectile tissue. The drug Viagra is effective owing to its potent and specific inhibition of a cGMP-phosphodiesterase (enzyme responsible for converting cGMP to GMP) isozyme that is expressed in the male reproductive organ.

The $\mathrm{Ca}^{2+}$ ion is also widely recognized as having an important signal transduction role in all eukaryotic cells. $\mathrm{Ca}^{2+}$ functions as a second messenger that can transduce extracellular stimuli such as light, hormones, nervous impulses, or stress to produce a coordinated and appropriate metabolic response. In response to an external stimulus the rate at which $\mathrm{Ca}^{2+}$ ions are transported into or out of a specific subcellular compartment is modified. The resulting change in intracellular free $\mathrm{Ca}^{2+}$ concentration represents the "signal," which elicits appropriate metabolic responses, sometimes as a direct effect of $\mathrm{Ca}^{2+}$ on the target enzyme and sometimes via stimulation of a protein kinase that then phosphorylates the target enzyme. It is generally accepted that animal and plant cytosolic free $\mathrm{Ca}^{2+}$ concentrations are maintained in the range of 0.1 to $1 \mu \mathrm{M}$ and that metabolic processes in the cytosol are activated by an increase in free $\mathrm{Ca}^{2+}$ concentration from 0.5 to $5 \mu \mathrm{M}$. The very low cytosolic $\mathrm{Ca}^{2+}$ concentration is maintained via the active transport of $\mathrm{Ca}^{2+}$ ions by various membrane-localized $\mathrm{Ca}^{2+}$ pumps that move the ion against its concentration gradient to return it to the extracellular space or to various subcellular compartments (i.e., the lumen of the endoplasmic reticulum). Several types of specific $\mathrm{Ca}^{2+}$ channels (that facilitate $\mathrm{Ca}^{2+}$ influx) and ATP-dependent pumps (for mediating $\mathrm{Ca}^{2+}$ efflux) are present in membranes of animal and plant cells and are tightly regulated by plasma-membrane-associated and intracellular control mechanisms. Thus, eukaryotic cells are endowed with a complex "machinery" with which to maintain or alter $\mathrm{Ca}^{2+}$ concentration gradients.

Many of the effects of $\mathrm{Ca}^{2+}$ on animal metabolism are mediated by $\mathrm{Ca}^{2+}$ acting through the highly conserved $\mathrm{Ca}^{2+}$-binding protein, calmodulin, to activate $\mathrm{Ca}^{2+} /$ calmodulin-dependent protein kinases. There is ample evidence that plants also utilize $\mathrm{Ca}^{2+}$ and calmodulin to mediate protein phosphorylation, and that this is an important mechanism whereby various extracellular stimuli are coupled to intracellular metabolic events. Relative to animal systems, however, far less is known about the structure or control of plant protein kinases. A number of $\mathrm{Ca}^{2+}$-dependent and calmodulin-independent protein kinases, which appear to be unique to the plant kingdom, have been purified and cloned from various plant sources. The enzymes contain both a protein kinase catalytic domain and a $\mathrm{Ca}^{2+}$-binding domain similar to calmodulin. This explains the direct activation of the enzyme by $\mathrm{Ca}^{2+}$ and has established it as the prototype for a new class of protein kinase.

\section{Protein Kinases That Respond to Intracellular Signals}

Most kinases described to date respond to extracellular signals. However, there are at least two categories of protein kinases that respond to intracellular signals such as changes in AEC or concentrations of metabolic intermediates.

The mammalian AMP-dependent protein kinase (AMPK) was initially discovered as a regulator of various key pacemaker enzymes of anabolic and catabolic pathways. As previously discussed, due to the action of adenylate kinase, a sharp elevation in AMP concentration occurs whenever the cell is subjected to stresses that cause ATP depletion, for example, hypoxia, heat shock, glucose starvation, and (in muscle) exercise. The rapid rise in AMP activates AMPK and leads to the phosphorylation of target enzymes. Thus, the AMPK cascade acts as an intracellular "fuel gauge," monitoring the AEC of the cell. Once activated by a decrease in AEC, AMPK acts to conserve 


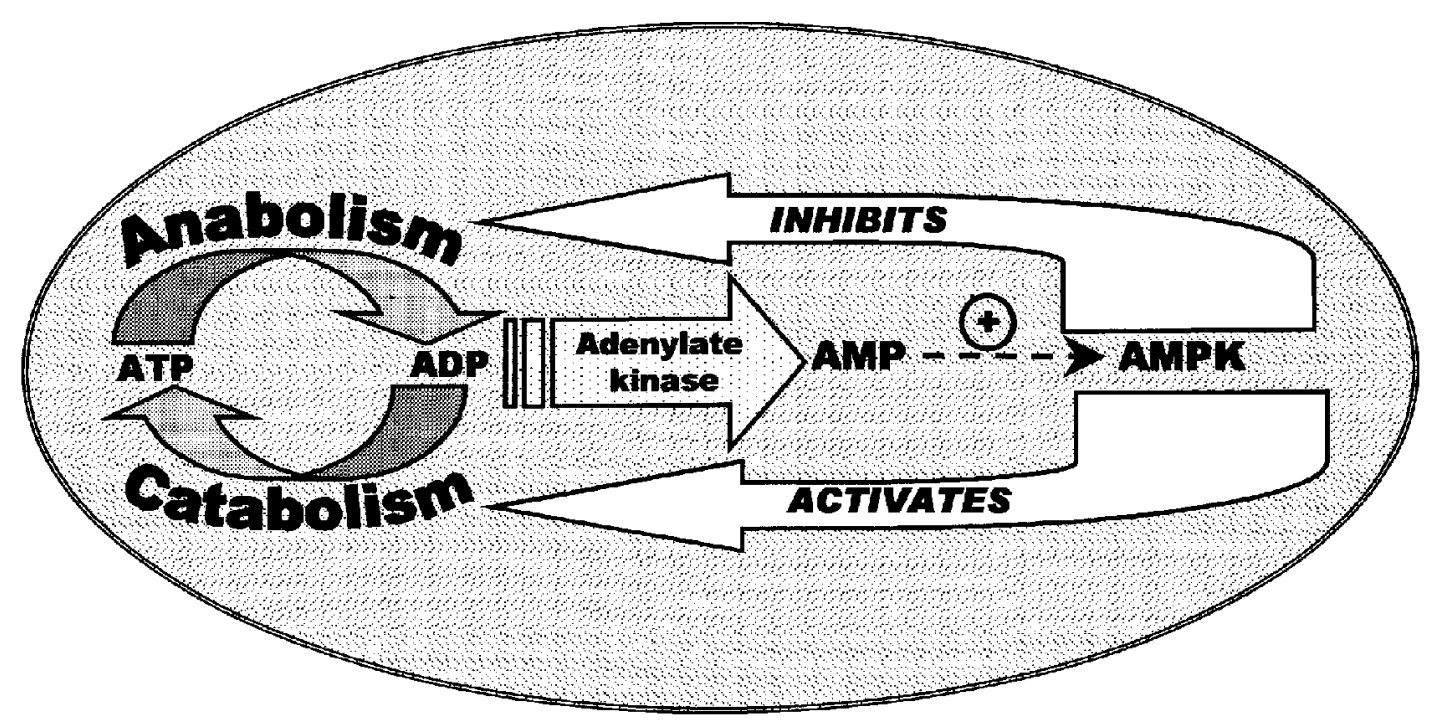

Figure 1.8 A general model for the role of the AMP-activated protein kinase (AMPK) as an "energy charge" monitor within the cell. If cellular stress causes the rate of an ATP-consuming pathway to exceed that of an ATP-producing pathway, adenylate kinase will catalyze the net conversion of ADP to AMP, which activates the AMPK. AMPK then catalyzes the phosphorylation of key enzymes of anabolism and catabolism.

ATP by inhibiting key enzymes of ATP-consuming anabolic pathways and promoting ATP production by stimulating key enzymes of ATP-producing catabolic pathways such as those involved in glucose uptake, glycolysis, and fatty acid oxidation (Fig. 1.8). AMPK is activated in two ways by AMP: first, AMP is an allosteric activator of the enzyme, and, second, AMP makes the enzyme more susceptible to activation via phosphorylation by another protein kinase (called a kinase kinase). The combined effects cause an up to 50-fold increase in the activity of AMPK in response to elevated AMP, providing further amplification of the effect of a reduction in ATP levels. AMPK now appears to be one of the primary intracellular sensors of cellular AEC, and most effects of ATP depletion may actually be mediated through the AMPK pathway, rather than via direct allosteric effects of the adenine nucleotides on metabolic enzymes. The AMPK cascade appears to be highly conserved between vertebrates, yeast, and higher plants and may have evolved from an ancient "stress response" system.

Protein kinases that are allosterically controlled by intracellular metabolites tend to specifically phosphorylate a single target enzyme to which they are usually tightly bound. The specific phosphatases that dephosphorylate the phosphoenzyme are also tightly bound to the target enzyme. A good example of this is the pyruvate dehydrogenase complex (PDC) of eukaryotic mitochondria that catalyzes the key reaction (pyruvate $+\mathrm{CoA}+\mathrm{NAD}^{+}$ $\rightarrow$ acetyl-CoA $+\mathrm{NADH}+\mathrm{CO}_{2}$ ) that links the glycolytic pathway with the Krebs cycle. PDC is subject to inactivation when phosphorylated by a specific PDC kinase. The PDC kinase is allosterically activated by metabolites such as acetyl-CoA and NADH that accumulate when mitochondrial respiration is suppressed (i.e., during hypoxia) or when mitochondrial oxidative capacity is at its limit. Conversely, PDC kinase is inhibited by metabolites such as ADP that signal a need for more respiratory metabolism.

Phosphoprotein Phosphatases The action of protein kinases is reversed by phosphoprotein phosphatases that tend to have very broad substrate specificities, making determination of their physiological functions difficult. Phosphoprotein phosphatases have been classified into four main groups (Table 1.5). These phosphatases are generally highly conserved proteins that appear to be ubiquitous to all eukaryotic cells.

The activity of phosphoprotein phosphatase 1 is specifically inhibited by a regulatory protein termed inhibitor 1 , which complexes with the enzyme under conditions when the corresponding protein kinase is active. Inhibitor 1 only does so when it has been phosphorylated by protein kinase A. Thus, activation of protein kinase A leads to inhibitor-1 phosphorylation, which reduces the dephosphorylating activity of protein phosphatase 1 , and thereby augments the increase in target enzyme phosphorylation due to protein-kinase-A activity (e.g., see Fig. 1.6). Phosphoprotein phosphatase $2 \mathrm{~B}$ is activated by $\mathrm{Ca}^{2+}$ and 
TABLE 1.5 Classification of Major Phosphoprotein Phosphatases Important in Metabolic Control

\begin{tabular}{|c|c|c|}
\hline Type & Specific Properties & Typical Targets \\
\hline 1 & $\begin{array}{l}\text { a. Inhibited by phosphorylated inhibitor } 1 \\
\text { b. Particulate (i.e., bound to glycogen) }\end{array}$ & Glycogen metabolism, muscle contraction \\
\hline $2 \mathrm{~A}$ & Cytosolic & Glycolysis, gluconeogenesis, fatty acid synthesis \\
\hline 2B & $\begin{array}{l}\text { a. Activity-dependent on } \mathrm{Ca}^{2+} / \text { calmodulin } \\
\text { b. Poor activity with metabolic enzymes } \\
\text { c. May result in } \mathrm{Ca}^{2+} \text { attenuation of the influences of cAMP }\end{array}$ & $\begin{array}{l}\text { Protein kinases, protein phosphatases, and inhibitor } 1 \text { that } \\
\text { have been phosphorylated by protein kinase A }\end{array}$ \\
\hline $2 \mathrm{C}$ & $\begin{array}{l}\text { a. Cytosolic } \\
\text { b. } \mathrm{Mg}^{2+} \text { required as a cofactor }\end{array}$ & $\begin{array}{l}\text { AMP-dependent protein kinase, and possibly the targets } \\
\text { of AMP-dependent protein kinase }\end{array}$ \\
\hline
\end{tabular}

$\mathrm{Ca}^{2+} /$ calmodulin and specifically acts on the protein kinases, phosphatases, and inhibitor 1 that are phosphorylated by protein kinase $\mathrm{A}$ (and thus provides another link between the cAMP and $\mathrm{Ca}^{2+}$ signal transduction systems).

Disulfide-Dithiol Interconversion Reversible covalent modification by disulfide-dithiol exchange involves reactions that are chemically similar to those that occur in the formation of the disulfide bonds that stabilize the tertiary structure of certain proteins. However, unlike structural disulfide bonds, those that participate in the control of enzyme activity must be readily susceptible to reduction back to dithiol groups via the action of compounds such as reduced thioredoxin.

Thioredoxin is a $12-\mathrm{kDa}$ heat-stable protein that in its reduced $\mathrm{SH}$ form can function as a protein-disulfide reductase. Thioredoxin has been found in all cell types and all phyla where it has been sought. The protein plays a variety of different roles. In bacterial and animal systems it may function as a general protein-disulfide reductant, or it may participate in the reduction of specific enzymes. In these organisms reduced thioredoxin is regenerated by thioredoxin reductase, at the expense of NADPH (reduced NAD phosphate).

Disulfide-dithiol enzyme interconversion has a much greater regulatory significance in photosynthetic organisms. This type of modification is extremely important in linking photosynthetic electron transport flow with light control of several key chloroplastic enzymes involved in photosynthetic $\mathrm{CO}_{2}$ fixation and related processes. Reducing equivalents (electrons) are ultimately generated from water by the photosynthetic electron transport chain on the thylakoid membrane. The proteins ferredoxin, ferredoxin-thioredoxin reductase, and thioredoxin constitute the machinery that shuttles the reducing equivalents from the electron transport chain to selected target enzymes in the chloroplast stroma (Fig. 1.5b). Two different thioredoxins, designated $f$ and $m$ are part of the ferredoxin-thioredoxin system in chloroplasts. In the reduced state thioredoxin $f$ selectively activates several Calvin cycle enzymes involved in photosynthetic $\mathrm{CO}_{2}$ fixation, whereas thioredoxin $m$ preferentially activates and inhibits NADP-malate dehydrogenase and glucose-6-phosphate dehydrogenase, respectively. The thioredoxin system thus plays a key role in coordinating the light-dependent photosynthetic electron transport chain with the control of several key enzymes within the chloroplast stroma. By mechanisms not yet fully understood, enzymes that are reduced by thioredoxin in the light are oxidized to their disulfide forms in the dark. This might be catalyzed by the action of either oxidized thioredoxin or low-molecular-weight oxidants such as oxidized glutathione, dehydroascorbate, or hydrogen peroxide.

Fine Control 5. Subunit Association-Disassociation Usually, a multimeric enzyme becomes less active or inactive when its constituent subunits are dissociated. This property has been exploited to facilitate the control of certain enzymes. Aggregation or dissociation of subunits is normally induced by the binding of a small molecular weight allosteric effector molecule but may also be instigated by reversible covalent modification. For example, acetyl-CoA carboxylase is a tightly regulated enzyme that catalyzes the irreversible first committed step of fatty acid biosynthesis as follows: acetyl-CoA $+\mathrm{HCO}_{3}{ }^{-}+$ $\mathrm{ATP} \rightarrow$ malonyl-CoA $+\mathrm{ADP}+\mathrm{P}_{\mathrm{i}}$. In eukaryotic cells, the enzyme is subject to both allosteric regulation and reversible phosphorylation so as to control the flow of fatty acid precursors into malonyl-CoA. In its active, dephosphorylated form the enzyme's subunits polymerize into long filaments. As outlined in Figure 1.9, proteinkinase-mediated phosphorylation (triggered in vertebrates by the hormones glucagon or epinephrine) promotes dissociation of the acetyl-CoA carboxylase polymer into inactive monomeric subunits, thereby inhibiting fatty acid synthesis.

Another interesting example of enzyme control by subunit association-dissociation concerns the glycolytic enzyme, pyruvate kinase, from the mammalian pituitary gland. Researchers who isolated a clone for a thyroid 


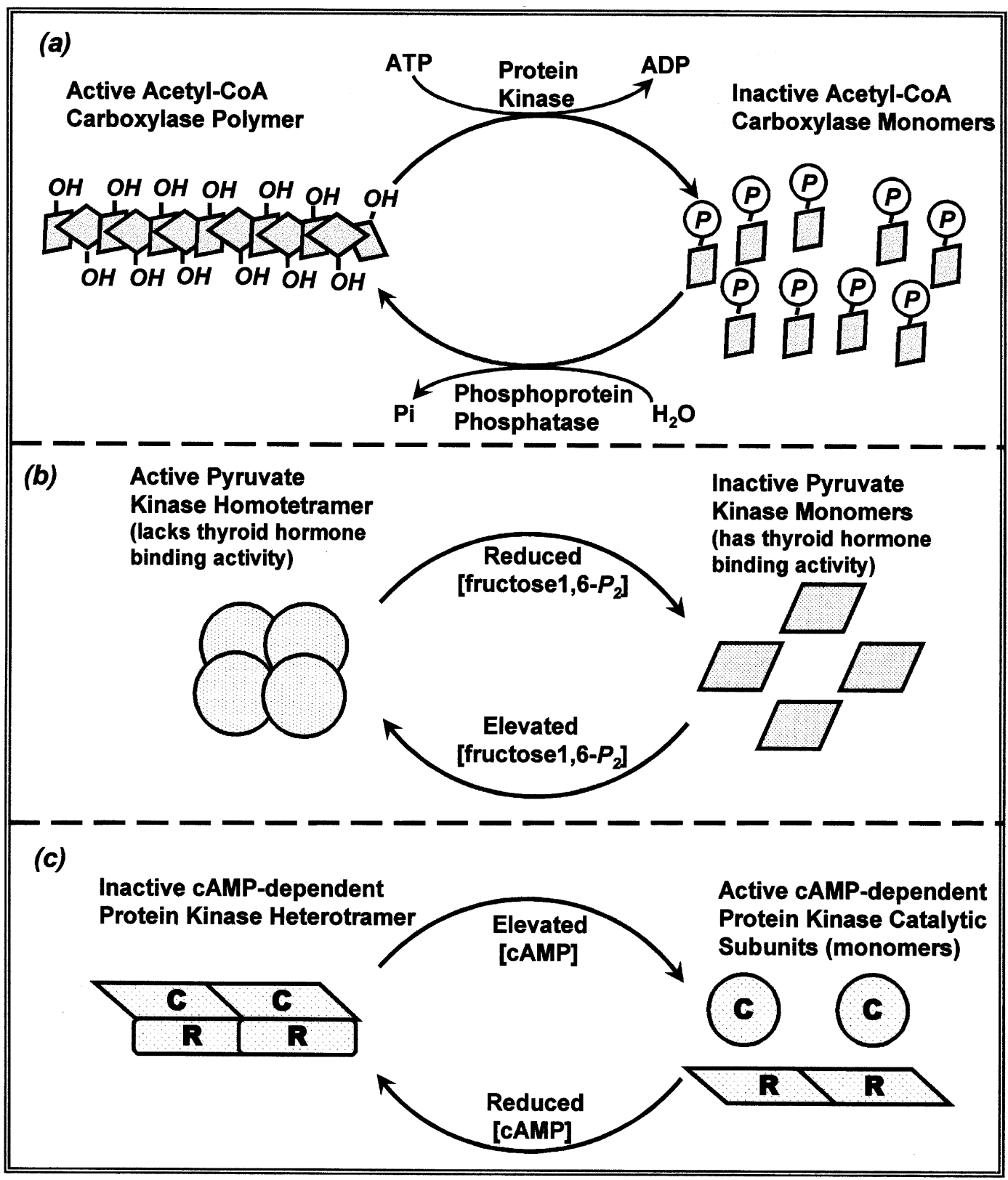

Figure 1.9 Examples of the fine control of enzyme activity by subunit association-dissociation. (a) Protein-kinase-mediated phosphorylation of mammalian acetyl-CoA carboxylase causes the enzyme to dissociate from its active polymeric form into inactive monomers. This process is reversed by the action of a phosphoprotein phosphatase. (b) Pyruvate kinase from pituitary glands dissociates from an active homotetramer into inactive monomers when levels of its allosteric activator (fructose-1,6-bisphosphate) are reduced, and vice versa. The inactive pyruvate kinase monomers acquire thyroid hormone binding activity. (c) In the inactive $\mathrm{R} 2 \mathrm{C} 2$ heterotetramer of cAMP-dependent protein kinase the regulatory subunits $(\mathrm{R})$ block the substrate binding sites of the catalytic subunits. (c) Cyclic AMP activates the enzyme by causing dissociation of the $\mathrm{C}$ subunits from the inhibitory $\mathrm{R}$ subunits. This allows the enzyme to phosphorylate various protein substrates that contain the cAMP-dependent protein kinase consensus sequence. 
hormone binding protein from a pituitary cDNA library unexpectedly discovered that its sequence exactly matched that previously reported for pituitary pyruvate kinase. Further research revealed that pituitary pyruvate kinase is a bifunctional protein that interconverts between an active pyruvate kinase tetramer (lacking thyroid hormone binding activity) and inactive pyruvate kinase monomers (having thyroid hormone binding activity) (Fig. 1.9). This interconversion has been attributed to the potent allosteric activator of pyruvate kinase, FBP. Elevated $[\mathrm{FBP}]$ promotes association of the monomeric subunits into the active pyruvate kinase homotetramer and reduced [FBP] promotes dissociation.

There are exceptions to the general rule that multimeric enzymes become less active when dissociated. For example, in its inactive state the cAMP-dependent protein kinase exists as a heterotetramer formed of two regulatory subunits (R) and two catalytic subunits (C). Allosteric activation by cAMP involves binding of four cAMP molecules by the two regulatory subunits and release of the free catalytic subunits (Fig. 1.9). The two sites for cAMP per regulatory subunit interact cooperatively so that the Hill coefficient for kinase activation with respect to cAMP is about 1.6. As previously discussed, this allows a more sensitive response by the enzyme to small changes in cAMP concentration than would be possible otherwise.

Fine Control 6. Reversible Associations of Sequential Enzymes The existence of known stable multienzyme complexes provides favorable evidence for the interaction of soluble enzymes that are metabolically sequential (i.e., that catalyze the consecutive reactions in a metabolic pathway). Due to high intracellular protein concentrations, such interactions are far more likely to occur in vivo than in the dilute solutions that are used for in vitro enzymological studies. The limited solvent capacity of the cell also supports the idea of interactions between sequential enzymes of a metabolic pathway since the need for a large pool of free intermediates would then be eliminated. There is now convincing evidence for subcellular structuring of metabolic pathways that were once believed to be entirely soluble. For example, various workers have described associations between many of the so-called soluble enzymes of glycolysis and membrane fractions or structural proteins in animal and plant cells. Of regulatory significance is the fact that the extent of enzyme binding appears to be closely related to pathway flux. During anaerobic muscle contraction, for instance, a marked increase in the binding of key glycolytic enzymes to contractile proteins has been observed. Enzyme binding dissipated once contractile activity, and need for a high glycolytic flux, ceased. Of equal significance are the findings that sequential glycolytic enzymes can also show specific interactions with each other-interactions that have mutual kinetic effects on enzyme activities. Similar correlations between the degree of glycolytic enzyme binding and rate of glycolytic flux have been observed in other animal and plant systems. Enzymes that reversibly bind to intracellular structures such as membranes or the cytoskeleton have been termed ambiquitous.

The microcompartmentation of enzymes and metabolic pathways that can result from ambiquitous interactions could provide an effective means of metabolic control via: (i) direct transfer or channeling of intermediates between consecutive enzymes (Fig. 1.10), and (ii) altering enzyme kinetic properties due to allosteric transitions occurring during binding. It has been suggested, therefore, that metabolic control of pathways such as glycolysis can occur not only by allosteric regulation and covalent modification of key enzymes but also by altering the partitioning of enzymes between the soluble and bound phases. The molecular mechanisms that control the extent of enzyme binding in vivo are not yet fully understood. Binding of enzymes in vitro can be influenced by $\mathrm{pH}$, concentrations of substrates, products and allosteric effectors, enzyme phosphorylation, and osmotic and/or ionic strength. For example, during muscle contraction PFK was found to be phosphorylated in vivo. However, there was no obvious influence of phosphorylation on the in vitro substrate saturation kinetics of PFK or its response to allosteric effectors. The physiological reason for covalent modification remained perplexing until it was discovered that PFK phosphorylation was a signal that promoted the association of the enzyme with contractile proteins (actin/myosin), and hence other metabolically sequential glycolytic enzymes.

Although there is compelling evidence for complex formation and/or binding of so-called soluble enzymes to structural elements in the cell and the direct transfer of substrates between sequential enzymes of animal cells, these possibilities have received relatively scant attention in plant systems. However, there is accumulating evidence that metabolically sequential enzymes in plant pathways may physically associate to form a multienzyme complex in vivo. For example, the chloroplastic ferredoxin, ferredoxin-thioredoxin reductase, and thioredoxin system used for disulfide-dithiol enzyme interconversion (Fig. 1.5b) may exist in the chloroplast stroma as an enzyme complex termed protein modulase. Protein-protein interactions may also play a significant role in addition to disulfide-dithiol exchange reactions in the light-dependent activation of several chloroplastic enzymes by thioredoxin. Various studies have also provided compelling evidence that there is direct channeling of substrates between Calvin cycle enzymes in illuminated chloroplasts and that the enzymes of this pathway exist as large supramolecular complexes that may reversibly associate with the thylakoid membrane in the light. 


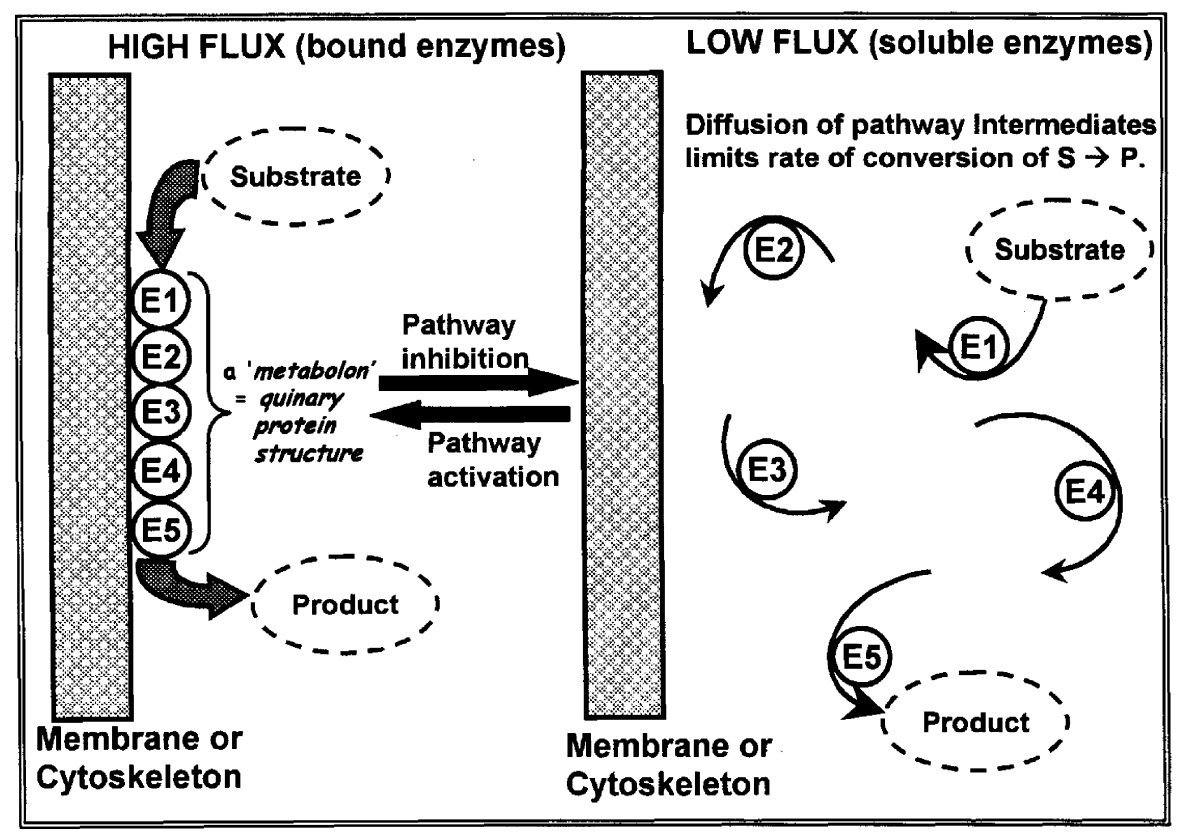

Figure 1.10 The control of enzyme activity by the reversible association of metabolically sequential enzymes into an organized multienzyme system, or metabolon (also termed quinary protein structure). E1, E2, E3, E4, and E5 represent the sequential enzymes that catalyze the consecutive reactions of a hypothetical metabolic pathway. Substrate (or S) and product (or P) depict the pathway's overall starting material(s) and end product(s), respectively.

The above studies indicate that before an overall understanding of metabolic control is achieved we must determine how, where, and when "soluble" metabolically sequential enzymes might be aggregated into microcompartments to form an organized multienzyme system or metabolon. It will also be of interest to determine how such associations may alter individual enzyme kinetic/ regulatory properties. Equally significant will be an analysis of the extent to which the reversible formation and dissolution of metabolons contributes to the overall integration and control of metabolism.

\section{CONCLUDING REMARKS}

Enzyme activity is one of the major factors influencing the magnitude of metabolic fluxes in any cell. Metabolic control may occur at several levels, beginning with the gene and proceeding through various stages of protein synthesis and turnover. More rapid alterations in metabolic flux occur through activation and inhibition of key enzymes along the major metabolic pathways, particularly by mechanisms such as reversible covalent modification and by the actions of effector molecules that reflect the cell's AEC, oxidation/reduction potential, and/or the accumulation of metabolic end products. Discoveries concerning metabolic control continue to be made at a rapid rate, particularly in the field of signal transduction. Each discovery adds to the view that signal transduction and metabolic control networks have remarkable intricacy. Although great advances have been made in understanding the mechanisms that contribute to the control of enzyme activity, our comprehension of why metabolic systems behave as they do in vivo is incomplete. However, the tools to address these questions are rapidly evolving, and advances in the theory of metabolic control and in computing power to analyze metabolism have kept pace with experimental developments. This holds great promise for those molecular geneticists who wish to reap a bounty via the process of metabolic engineering.

\section{LIST OF ABBREVIATIONS}

AEC adenylate energy charge

AMPK AMP-activated protein kinase

cAMP $\quad 3^{\prime}, 5^{\prime}$-cyclic adenosine monophosphate

cGMP $\quad 3^{\prime}, 5^{\prime}$-cyclic guanosine monophosphate

CoA coenzyme A

F6P fructose-6-phosphate 


$\begin{array}{ll}\text { FBP } & \text { fructose-1,6-bisphosphate } \\ K_{\text {eq }} & \begin{array}{l}\text { equilibrium constant for a chemical reaction } \\ \text { activation constant }=\text { activator concentra- } \\ K_{a}\end{array} \\ K_{m} \text { or } S_{0.5} & \begin{array}{l}\text { sion yielding half-maximal activation } \\ \text { enzymes that show hyperbolic or sigmoidal } \\ \text { substrate saturation kinetics, respectively }\end{array} \\ & \text { metabolic control analysis } \\ \text { MCA } & \text { Hill coefficient } \\ n_{H} & \text { nuclear magnetic resonance } \\ \text { NMR } & \text { substrate concentration } \\ \text { [S] } & \text { phosphoenolpyruvate } \\ \text { PEP } & \text { pyruvate dehydrogenase complex } \\ \text { PDC } & \text { intracellular pH } \\ \text { pH } & \text { inorganic phosphate } \\ \mathrm{P}_{\mathrm{i}} & \text { phosphofructokinase } \\ \mathrm{PFK} & \text { maximal rate of an enzyme-catalyzed } \\ V_{\mathrm{max}} & \text { reaction } \\ V_{0} & \text { initial rate of an enzyme-catalyzed reaction }\end{array}$

\section{REFERENCES}

ApRees, T., and Hill, S. A. (1994). Metabolic control analysis of plant metabolism. Plant, Cell Environ 17:587-599. A clearly written and concise introduction to the concepts underlying metabolic control analysis, focusing on its application to plant metabolism.
Atkinson, D. E. (1977). Cellular Energy Metabolism and Its Regulation. Academic, New York. A classic book introducing the concept of adenylate energy charge.

Cornish-Bowden, A. (1999). Fundamentals of Enzyme Kinetics. Portland Press, London. A discussion of the principles of enzyme kinetics, with an emphasis on principles rather than an accumulation of facts. More advanced.

Fell, D. A. (1997). Understanding the Control of Metabolism. Portland Press, London. An appealing introduction to the basic theories of metabolism and metabolic control analysis. More advanced.

Knowles, V. L., Smith, C. S., Smith, C. R., and Plaxton, W. C. (2001). Structural and regulatory properties of pyruvate kinase from the cyanobacterium Synechococcus PCC 6301. J Biol Chem 276:20966-20972.

Plaxton, W. C. (1996). The organization and regulation of plant glycolysis. Annu Rev Plant Physiol Plant Mol Biol 47:185214. Helpful review on glycolytic compartmentation and control in plants. Compares and contrasts plant and animal glycolysis.

Price, N. C., and Stevens, L. (1989). Fundamentals of Enzymology, 2nd ed. Oxford University Press, New York. Excellent book that provides a comprehensive and readable account of enzyme properties in systems of increasing complexity (from purified enzymes to enzymes in the living cell).

Thomas, S., and Fell, D. A. (1998). The role of multiple enzyme activation in metabolic flux control. Advan. Enzyme Regul. 38: $65-85$. 